\title{
Advances in Hollow Inorganic Nanomedicines for Photothermal-Based Therapies
}

This article was published in the following Dove Press journal:

International Journal of Nanomedicine

\author{
Chen Ling' \\ Xiaobo Wang $\mathbb{D}^{\prime}$ \\ Yan Shen $\mathbb{D}^{2}$ \\ 'School of Pharmacy, China \\ Pharmaceutical University, Nanjing \\ 2 III00, People's Republic of China; \\ ${ }^{2}$ Department of Pharmaceutics, China \\ Pharmaceutical University, Nanjing \\ 21 I 100, People's Republic of China
}

\begin{abstract}
Nanotechnology has prompted the development of hollow inorganic nanomedicine. These medicines are now widely investigated as photothermal-based therapies for various diseases due to their high loading capacity, tuneable wavelength, relatively small size and low density. We begin this review with a brief introduction, followed by a summary of the development of imaging-guided photothermal therapy (PTT) for cancer treatment during the last three years (from 2017 to 2020). We then introduce the antibacterial effects of these medicines on some bacterial infections, in which the pathogenic bacteria can be killed by mild photothermal effects, ions and antibiotic release. Other diseases can also be treated using hollow inorganic photothermal agents. Specifically, we discuss the use of PTT for treating Alzheimer's disease, obesity and endometriosis. Finally, we share our perspectives on the current challenges and future prospects of using hollow inorganic materials in clinical PTT for various diseases.
\end{abstract}

Keywords: hollow inorganic nanomedicine, photothermal therapy, diseases

\section{Introduction}

In the modern world, cancer is a predominant cause of death and is a very serious and challenging health problem currently faced by humanity. A recent study projected that over 1.8 million new cancer cases and over 0.6 million cancer deaths would occur in the US alone in $2020 .^{1}$ However, with the advancement of medical technology, many cancer therapies have been developed in the last 20 years, including surgery, radiotherapy and chemotherapy, ${ }^{2,3}$ resulting in a continuous decline in the mortality rate of cancer. ${ }^{1}$ However, there are still some drawbacks to the clinical application of these therapies, including recurrence, non-selective targeting, low therapeutic indices, multiple drug resistance and serious side effects. ${ }^{4,5}$ In addition, bacterial infections are a serious health problem that account for almost one-third of global mortality ${ }^{6}$ and considerable financial losses. ${ }^{7}$ Antibiotics are the most effective and frequently used treatments for bacterial infections, but the overuse of antibiotics in the clinical setting has contributed to pathogenic resistance. ${ }^{8,9}$ Multidrug resistance (MDR) is commonly seen in the context of both cancer and antibacterial therapies. Therefore, novel therapeutic strategies are urgently needed for combating both cancer and bacterial infections.

Recently, photothermal therapy (PTT) has been developed as a novel hyperthermia-based disease treatment strategy, in which photo-sensitive photothermal agents (PTAs) delivered at target sites in the body are used to convert near-infrared (NIR) light to heat in order to induce local hyperthermia ${ }^{10,11}$ The photothermal effect can
Correspondence: Yan Shen Tel +86 (025) 83271305

Email shenyan@cpu.edu.cn
International Journal of Nanomedicine 2021:16 493-513 
ablate aberrant cells and pathogenic bacteria, denaturing their proteins and causing cell death. ${ }^{6,12-15}$ This laserinduced hyperthermia therapy is a robust and efficient therapeutic strategy for disease treatment, with the merits of high selectivity, ${ }^{16}$ relatively low rates of side effects ${ }^{11}$ and negligible invasiveness. ${ }^{17}$ Due to these beneficial features, PTT is believed to be a promising strategy for treating various diseases. Meanwhile, PTT is also deemed as a good helper for other therapies, more and more synergistic therapies combining PTT and other therapies such as chemotherapy, ${ }^{18}$ photodynamic therapy, ${ }^{19}$ immunotherapy ${ }^{20}$ and starvation therapy ${ }^{21}$ are developed for cancer treatment. For example, the effect of chemotherapy can be enhanced and the multidrug resistance can be reduced by using a chemo/photothermal synergistic therapy; moreover, the photothermal effect can also contribute to the targeted drug release from nano-carrier at tumor site. In other words, the synergistic therapies are more than simply putting two therapies together, the introduction of PTT can significantly enhance the effect of other therapies.

To date, various types of therapeutic PTAs have been discovered, including inorganic types such as gold nanomaterials, $^{22}$ carbon-based nanomaterials, ${ }^{23}$ silica nanomaterials $^{24}$ and metal chalcogenides, ${ }^{25}$ and organic types such as conjugated polymers ${ }^{26}$ and porphysomes. ${ }^{27}$ Although organic nanomaterials are superior to their inorganic counterparts in terms of biocompatibility and biodegradability, they suffer from several limitations, such as unstable photothermal effects and low photothermal conversion efficiency (PCE). Owing to their excellent imaging capacity and PCE, inorganic PTAs are currently prioritised by researchers and have seen wide application in the diagnosis and treatment of diseases. Notably, the morphology of inorganic nanoscale PTAs exerts a great influence on their properties. Specifically, aggregates of nanoparticles (NPs) are usually limited by large size and instability, whereas solid NPs are limited by weak NIR absorption and narrow wavelength adjustability. ${ }^{28}$ Thus, the emergence of nanomaterials with hollow structures could provide a solution to these obstacles. By changing the diameter and thickness of the shell, the optical properties of hollow NPs can be easily manipulated, with the absorption wavelength ranging from near-ultraviolet (UV) to infrared. $^{29,30}$ In addition, hollow nanostructures possess lower mass than other nanostructures of the same size, thereby contributing to a relatively higher PCE per unit mass. $^{31}$ Moreover, the hollow interior endows hollow nanomaterials the capacity to be loaded with drugs, including imaging contrast agents (perfluorohexane for ultrasound [US] imaging) and therapeutic drugs (doxorubicin for cancer treatment), thus promoting both imaging and therapeutic efficacies. Owing to their features including high loading capacity, tuneable wavelength, relatively small size and low density, ${ }^{32,33}$ hollow inorganic nanostructures can be ideal agents in photothermal-based therapies for various diseases.

Reviews concerning the application of hollow or inorganic nanomedicines in PTT for cancer have been published. ${ }^{29,34-36}$ However, reviews of the application of both hollow and inorganic nanomedicines in cancer PTT have not been published in recent three years. Moreover, their applications to other noncancerous diseases have never been summarized in a review. In this review, we focus on the recent advances in the development of hollow inorganic nanomaterials for PTT-based treatments for cancer, bacterial infections and other diseases, including Alzheimer's disease (AD), obesity and endometriosis (Figure 1). We conclude with our perspectives on the current challenges and future prospects of using hollow inorganic materials in clinical PTT for various diseases.

\section{Photothermal-Based Therapy for Cancer Treatment Hollow Gold Nanostructures}

Due to special interactions with light, the free electrons of gold nanostructures undergo a collective coherent oscillation process known as localized surface plasmon resonance. ${ }^{15,37}$ Gold nanostructures can be endowed with optimal photothermal abilities, which are usually affected by the size, shape and dielectric constant of the nanostructure. ${ }^{38}$ In gold hollow nanostructures in particular, the photothermal effects are likely to be affected by the thickness of the shell. ${ }^{29}$ Recently, many hollow nanostructures have been developed for photothermal-based cancer therapy (Table 1). ${ }^{18,19,39-44}$

\section{Hollow Gold Nanospheres or Nanoshells}

Among all the hollow gold nanostructures, hollow gold nanospheres and nanoshells are frequently investigated. In 2003, Hirsch et al reported an SH-PEG-modified gold nanoshell for magnetic resonance (MR) temperature imaging-monitored photothermal therapy for transmissible venereal tumor. ${ }^{45}$ In that study, a PEG-passivated nanoshell was injected interstitially into the tumour site and exposed to NIR light $\left(820 \mathrm{~nm}, 4 \mathrm{~W} / \mathrm{cm}^{2}, 5-\mathrm{mm}\right.$ spot 


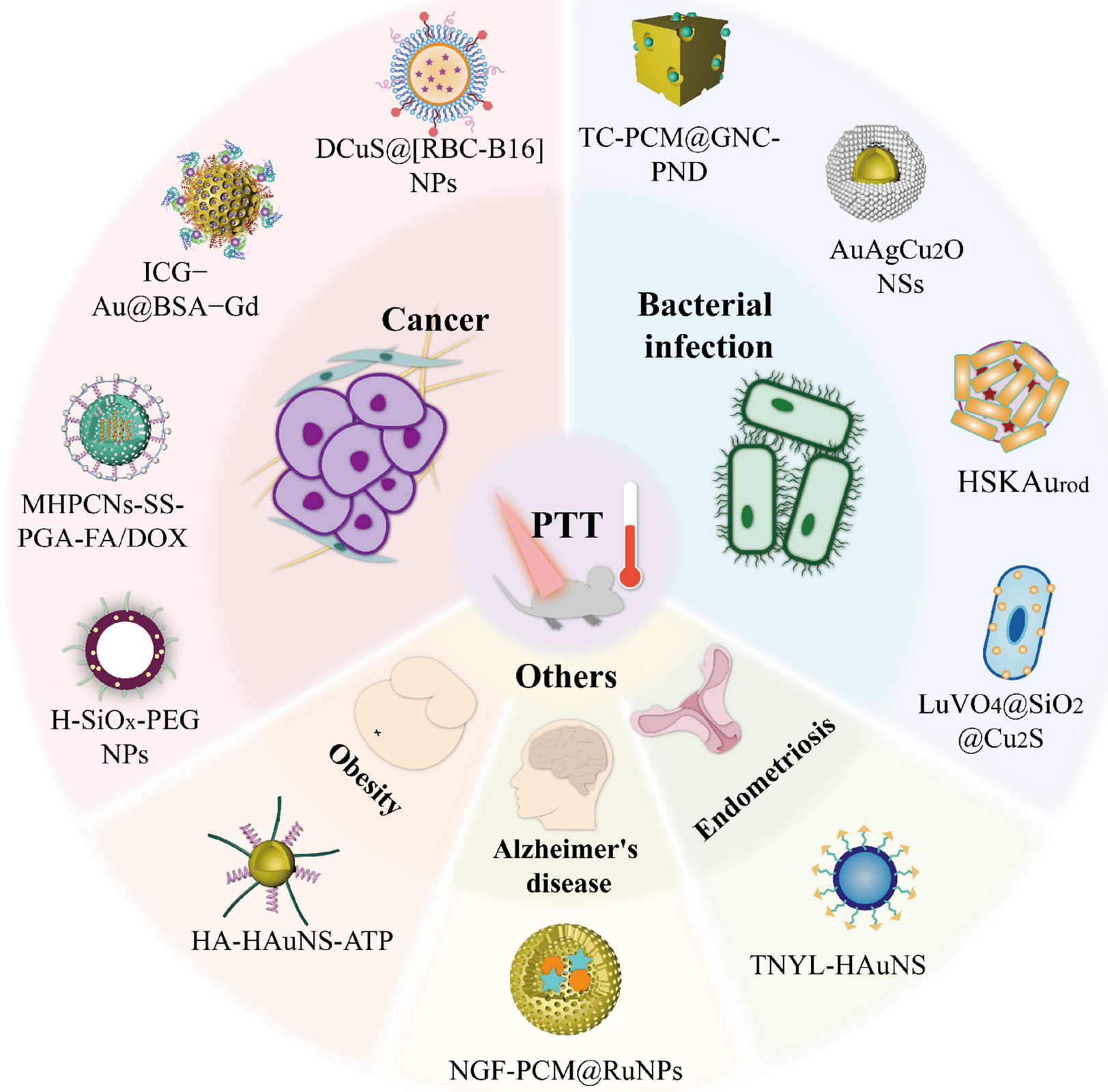

Figure I Schematic illustration for the PTT-based treatment in cancer, bacterial infections and other diseases including obesity, Alzheimer's disease and endometriosis. The outside hollow inorganic materials are examples for different diseases treatment.

diameter, $6 \mathrm{~min}$ ) for photothermal ablation under MRI monitoring. Fluorescence imaging based on the NIR lightabsorbing effects of hollow gold nanospheres (HAuNS) has also shown potential in cancer diagnosis. Wang et al developed an NIR fluorophore, cypate-conjugated HAuNS, for tumour-specific imaging and PTT. ${ }^{46}$ The fluorophore cypate was linked to HAuNS via a short spacer containing a urokinase-type plasminogen activator (uPA, an enzyme secreted by tumours) cleavable motif that could release the fluorophore to yield fluorescence in breast tumour cells. In 2017, You et al developed a hollow gold nanoshell (ICG-Au@BSA-Gd) for quadmodel NIR fluorescence/photoacoustic (PA)/computed tomography (CT)/MR imaging-guided photodynamic (PD) and photothermal synergistic therapy for breast cancer. ${ }^{19}$ In that study, the gold nanoshell was loaded with the common photosensitizer ICG to achieve photothermal (gold and ICG) and photodynamic (ICG) 


\begin{tabular}{|c|c|c|c|c|c|c|c|c|c|c|c|c|c|c|c|}
\hline 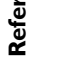 & $\underline{\underline{\underline{w}}}$ & $\Phi$ & $\bar{\Xi}$ & $\overline{\mathcal{g}}$ & $\overline{\underline{y}}$ & 导 & $\overline{\bar{n}}$ & $\widetilde{\widetilde{n}}$ & $\overline{\tilde{n}}$ & F & 氙 & 宫 & $\stackrel{\bar{\infty}}{\mathscr{D}^{\circ}}$ & $\overline{\overline{0}}$ & $\underset{\mathscr{D}}{\widetilde{D}}$ \\
\hline 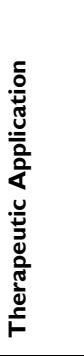 & 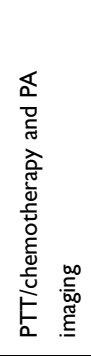 & 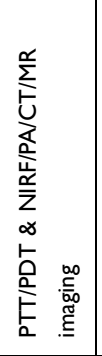 & 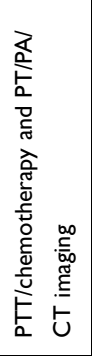 & 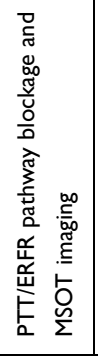 & 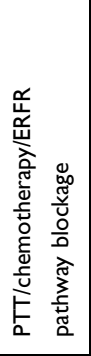 & 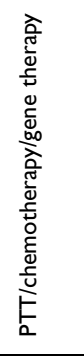 & 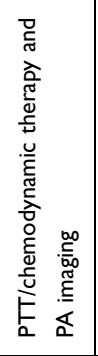 & 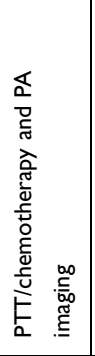 & 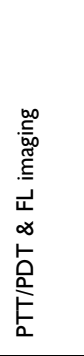 & 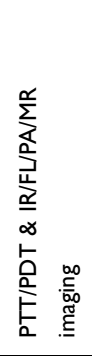 & 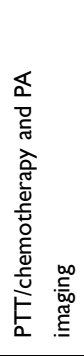 & 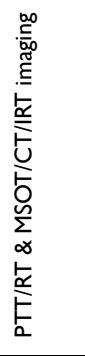 & 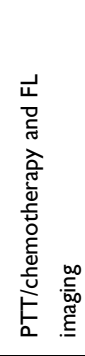 & 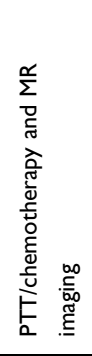 & 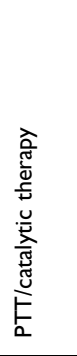 \\
\hline 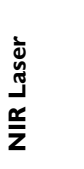 & 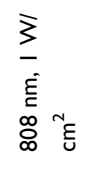 & 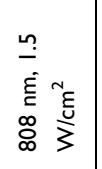 & 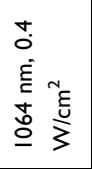 & 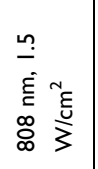 & 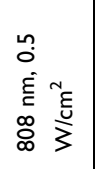 & 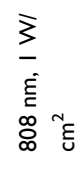 & 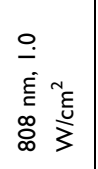 & 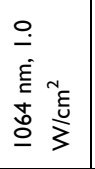 & 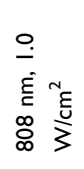 & 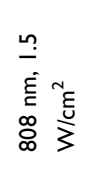 & 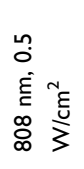 & 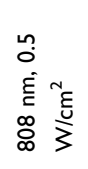 & 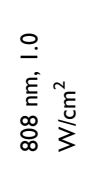 & 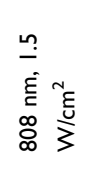 & 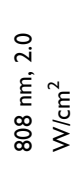 \\
\hline 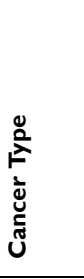 & 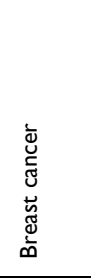 & 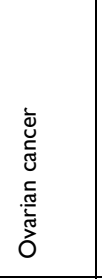 & 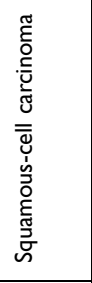 & 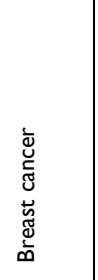 & 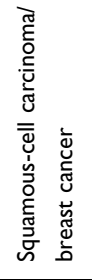 & 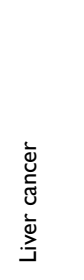 & 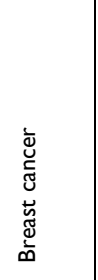 & 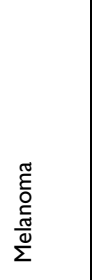 & 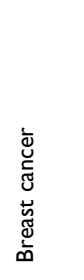 & 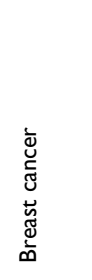 & 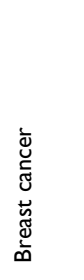 & 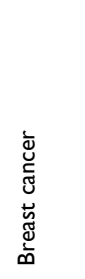 & 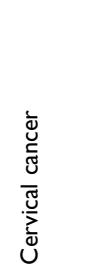 & 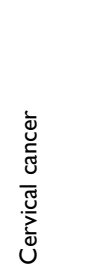 & 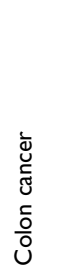 \\
\hline 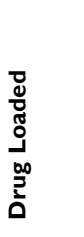 & 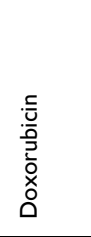 & 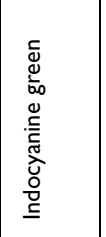 & 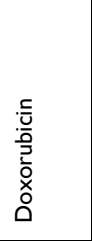 & 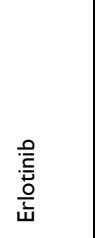 & 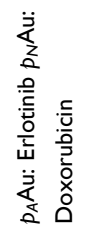 & 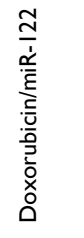 & 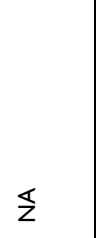 & 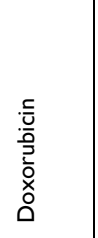 & ֻّ & Ů & 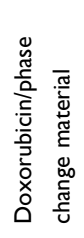 & 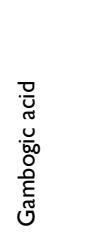 & 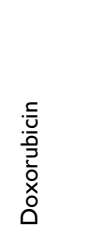 & 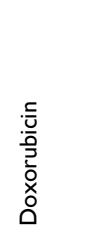 & 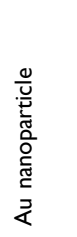 \\
\hline 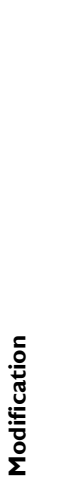 & 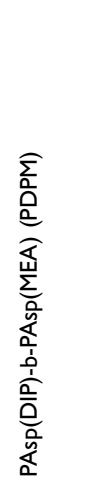 & 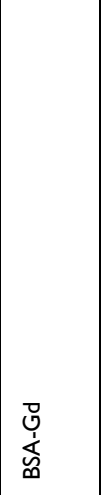 & 总 & 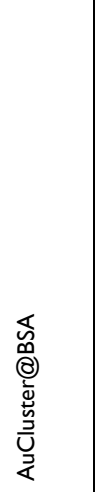 & 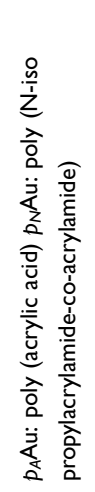 & 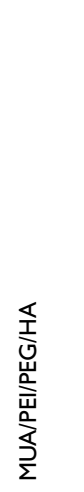 & $\mathbb{Z}$ & 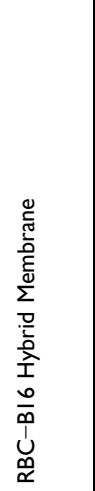 & 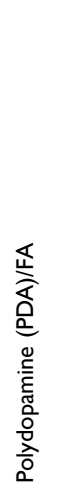 & 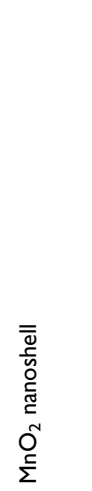 & $\mathbb{Z}$ & $\overleftrightarrow{I}$ & 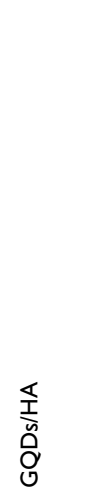 & 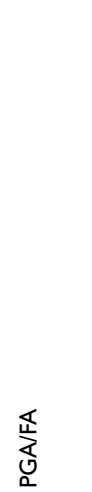 & $\mathbb{Z}$ \\
\hline 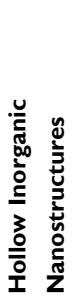 & $\begin{array}{l}\text { U } \\
\text { Ò } \\
\vdots \\
\Delta\end{array}$ & 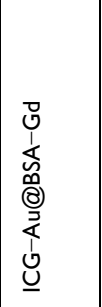 & 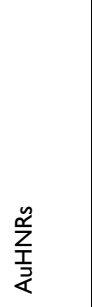 & 变 & 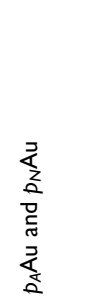 & 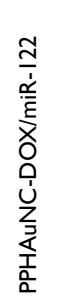 & 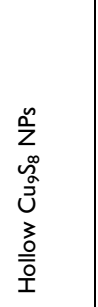 & 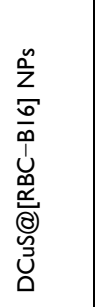 & 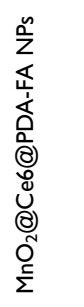 & 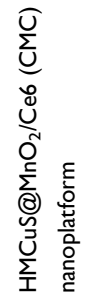 & $\begin{array}{l}\text { 吅 } \\
\text { (ृ) } \\
\text { 口ิ }\end{array}$ & 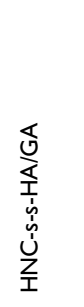 & 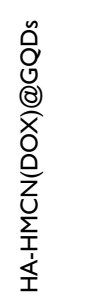 & 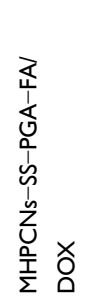 & 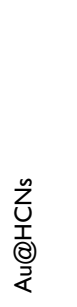 \\
\hline & $\begin{array}{l}\frac{7}{0} \\
0\end{array}$ & & & & & & 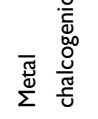 & & & & & & $\begin{array}{l}\delta \\
\text { o̊ } \\
\text { J }\end{array}$ & & \\
\hline
\end{tabular}




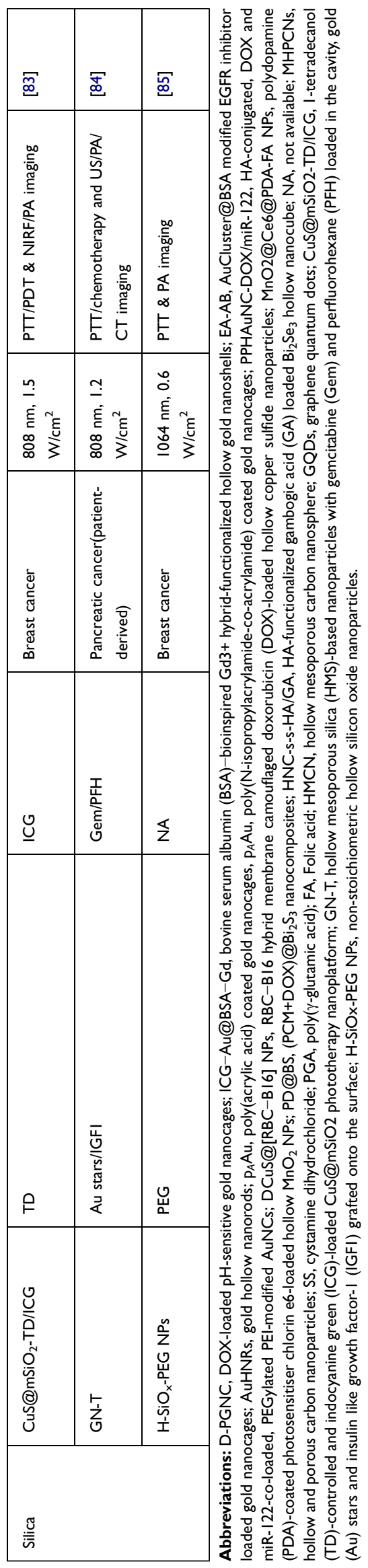

synergistic therapy under NIR light irradiation. As an FDA approved small molecule, ICG is widely applied in imaging or therapies of cancer. As mentioned, ICG can be solely used to achieve fluorescent and photoacoustic imaging and PTT/PDT. However, it is easily to aggregate and degrade in aqueous solution such as plasma, thus like many other small molecular drugs, the protection of ICG is usually carried out by nanoparticles encapsulation. In addition, this was the first study to synthesize a gadolinium (Gd)-based bovine serum albumin (BSA-Gd) hybrid to improve Gd-loading capacity, which endowed NPs with excellent MR/CT/PA imaging capacity. Coating with BSA guaranteed the biocompatibility and stability of the NPs. In subsequent in vivo experiments, quad model imaging was shown to be a precise and efficient method for the guidance of combined PTT and PDT.

\section{Hollow Gold Nanocages}

As another member of the hollow gold nanostructure (HGN) family, the biological application of gold nanocages, especially in cancer treatment, has been widely investigated. With their hollow interior and porous walls, ${ }^{4,48}$ gold nanocages are suitable for drug delivery, imaging and PTT. ${ }^{49}$ In 2018, Zhao et al developed gold nanocages (AuNCs) for melanoma-targeted positron emission tomography/CT imaging with radiolabelled ${ }^{64} \mathrm{Cu} .{ }^{50}$ The targeting ability stemmed from the conjugated $\alpha$ melanocyte-stimulating hormone $(\alpha-\mathrm{MSH})$ peptide, which could specifically bind to the over-expressed melanocortin 1 receptor in tumour cells. Moreover, the targeting efficiency was improved by increasing the amount of $\alpha-\mathrm{MSH}$ loaded on the AuNCs. Zhou et al also investigated a PA imaging-guided $\mathrm{pH}$-sensitive gold nanocage (D-PGNC) for ovarian cancer PTT and the selective delivery of DOX to tumour cells, in which the $\mathrm{pH}$ value is lower than that in other cells. ${ }^{18}$ The nanocage acted as a cargo and heat producer under NIR light, contributing to PTT and PA imaging. Inside the nanocage, DOX was covered by a $\mathrm{pH}$-sensitive copolymer (PDPM) which released DOX at a low pH. Recently, Zhan et al developed a gold nanocluster-modified gold nanocage-based hybrid nanodrug $^{42}$ for multispectral optoacoustic tomography (MSOT) imaging-guided PTT for breast cancer and epidermal growth factor receptor (EGFR) pathway blockage. The nanodrug consisted of an EGFR inhibitor (EB)-loaded gold nanocage moiety and a gold nanocluster moiety. The former (gold nanocage moiety) acted as a drug carrier, MSOT imaging agent (functioned in sensing the 
nanodrug's biodistribution and metabolic process) and photothermal generator. While the latter one (gold nanocluster moiety) was designed for stabilisation, fluorescence detection (an indication of drug release) and EB release.

\section{Hollow Gold Nanorods}

As a novel type of hollow structure, gold hollow nanorods (AuHNRs) were fabricated for the first time in $2018 .^{41}$ In the synthesis section, researchers utilized Se-doped Te nanorod as a template, followed by the modification of agents with sulfhydryl. The aspect ratio of AuHNRs was only 3, which endowed them with LSPR peak in NIR- II region. Then, under $1064 \mathrm{~nm}$ laser irradiation, a multimodel photothermal/photoacoustic/computed tomography imaging could be realized. With the hollow structure, AuHNRs were also used for drug-loading, and achieved targeted chemo/photothermal therapy in mouse model of squamous-cell carcinoma.

\section{Hollow Metal Chalcogenides}

Recently, metal chalcogenides have emerged as a novel tool for imaging-guided PTT. Examples include copper sulphide nanostructures, cobalt sulphide nanostructures, bismuth chalcogenides and manganese oxide nanostructures (Table 1). ${ }^{51-56}$ Similar to HGNs, hollow copper sulphide (CuS) NPs possess excellent NIR lightabsorbing capacity, resulting in high light-to-heat transformation efficiency. However, unlike HGNs, the optical absorption of CuS NPs does not stem from surface plasmon resonance, but rather from the $\mathrm{d}$-d bond transition of $\mathrm{Cu}$ ions, which is not affected by the size or shape of $\mathrm{CuS}$ NPs or the solvent. ${ }^{57}$ Moreover, HGNs are inferior to hollow $\mathrm{CuS}$ NPs in terms of biodegradability, toxicity and cost. ${ }^{58,59}$ As mentioned above, toxicity is a persistent problem with HGNs. However, due to their biodegradability, CuS NPs have lower toxicity and are more suitable for biological application. In 2019, Qiu et al developed the first photodegradable CuS NPs for residual tumour surface-enhanced Raman scattering (SERS) imaging and PTT. $^{60}$ Under exposure to NIR light, the hollow $\mathrm{CuS}$ NPs disseminated into tiny clusters, thereby contributing to their clearance from tissues after imaging and preventing chronic toxicity. Furthermore, given that the photothermal efficiency is not affected by the shape and size of $\mathrm{CuS}$ NPs, they may serve as a robust nanoplatform for prostate tumor PTT. Zhang et al fabricated hollow $\mathrm{CuS}$ nanoflower for MRI-guided DOX-loaded chemo/ photothermal liver cancer therapy. ${ }^{61}$ In their study, both $\mathrm{T}_{1}$-weighed MRI and $\mathrm{T}_{2}$-weighted fluid-attenuated inversion recovery ( $\mathrm{T}_{2}$-FLAIR) MRI were effective for imaging in in vitro experiments. However, the latter technique was more robust than the former; therefore, T2-FLAIR MRI was utilised for subsequent in vivo experiments. Wang et al utilised hybrid membrane-coated DOX-loaded CuS NPs (DCuS) for homotypic-targeting chemo-PTT with a long circulation life. ${ }^{52}$ As shown in Figure 2, the hybrid membrane was composed of membranes from both red blood cells (RBCs) and melanoma cells (B16-F10 cells). The former helped DCuS NPs to evade capture by the immune system, whereas the latter helped DCuS NPs to homogeneously target melanoma cells due to the expression of surface adhesion molecules. The combination of these two membranes (namely, RBC-B16) prolonged the circulation life of the nanomedicine.

It is worth noting that the clinical application of $\mathrm{CuS}$ nanostructures has a number of limitations. First, the PCE of CuS NPs is relatively low. Thus, PTT agents with a high PCE are urgently needed. Second, some CuS NPs are not effective at low concentrations. Therefore, highconcentration $\mathrm{CuS}$ NPs are generally used to achieve PTT. The excessive amount of $\mathrm{Cu}$ used results in the overexpression of vascular EGF, followed by the formation of more microvessels and the activation of pro-tumour pathways, resulting in the promotion of cancer growth. ${ }^{62-64}$ To tackle this problem, Guan et al designed hollow porous cobalt sulphide nanospheres (PCSH NSs) for MRI-guided PTT with an unprecedented high PCE of $70.1 \%$ compared to existing binary chalcogenides, which made PTT possible even at a low PTT agent concentration. ${ }^{65}$ In this study, the authors synthesized the nanostructures via a facile one-pot solvothermal synthetic method, where the molar ratio of thioacetamide to cobalt acetate was of vital significance as it was a decisive factor in its morphology. As the molar ratio was increased, the sizes of the nanostructures decreased, followed by the transformation from large nanosheets $($ molar ratio $=1)$ to nanospheres (molar ratio $=4$ ) and finally to solid nanostructures (molar ratio $=20$ ). The authors also proved that the concentration of $\mathrm{S}$ anions could affect the optical properties. Finally, in in vivo experiments, cervical tumour were eradicated with low doses of PCSH nanostructures (25 ppm) under NIR light ( $808 \mathrm{~nm}, 0.7 \mathrm{~W} / \mathrm{cm}^{2}$ ), with the lower chronic toxicity of nanomaterials contributing to fewer side effects. Unlike the biodegradable materials 


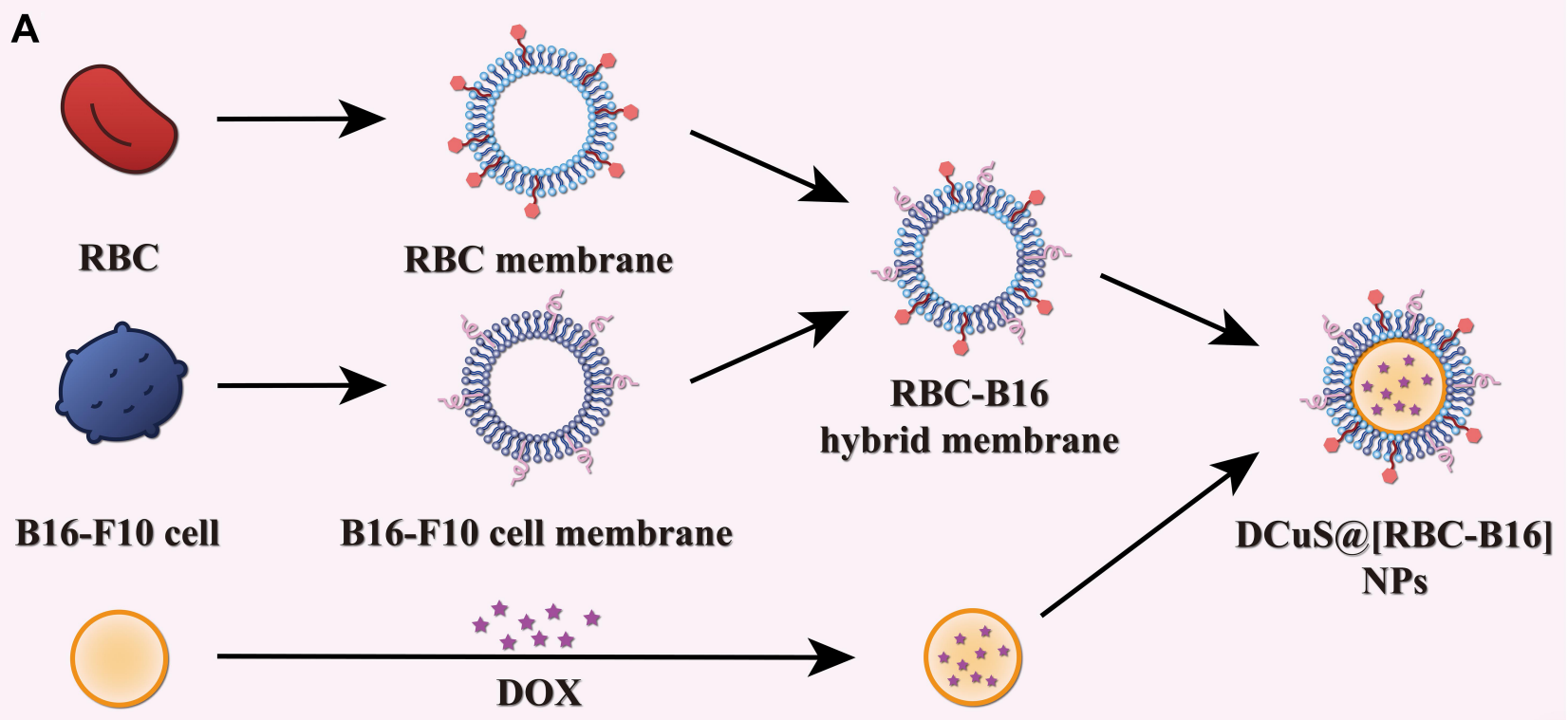

CuS NPs

DCuS NPs

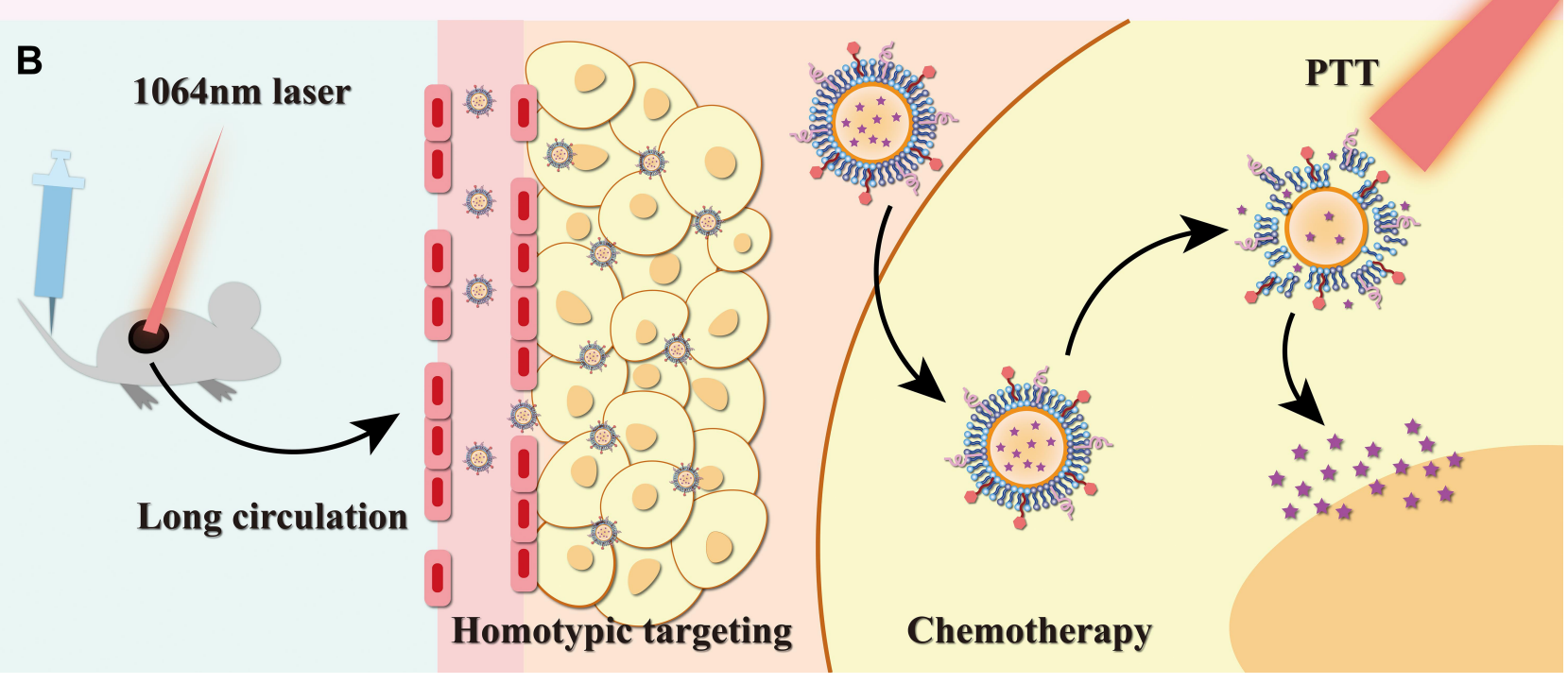

Figure 2 (A) Schematic of membrane fusion and coating. Membrane materials are derived from RBCs and B/6-FI0 cells and then fused together. The resulting hybrid membrane is used to camouflage DOX-loaded hollow copper sulfide nanoparticles (DCuS NPs) to produce DCuS@[RBC-BI6] NPs. (B) Synergistic photothermal/ chemotherapy of melanoma. Data from Wang et al. ${ }^{52}$

approach mentioned in the last paragraph, ${ }^{60}$ this approach was a more direct way of reducing chronic toxicity.

Manganese oxide, an excellent MRI contrast agent, has been widely used in MRI. ${ }^{6}$ In 2008, An et al designed hollow manganese oxide (MnO) NPs of different sizes for use in MRI. ${ }^{67}$ The authors found that the $\mathrm{T}_{1}$ relaxation was enhanced with increasing surface area, and that the hollow structures had a drug-loading capacity. In 2009, Shin developed hollow manganese oxide $\left(\mathrm{Mn}_{3} \mathrm{O}_{4}\right)$ NPs for MRI and drug loading. ${ }^{68}$ In another study, US imaging was combined with MRI for cancer imaging using some manganese oxide-based NPs. ${ }^{69}$ Although manganese oxide nanosheets $^{70}$ and mesoporous NPs ${ }^{71}$ have been previously applied for PTT, hollow $\mathrm{MnO}_{2}$ NPs have only recently been used. For the first time, Zeng et al fabricated polydopamine (PDA)-coated photosensitiser chlorin e6-loaded hollow $\mathrm{MnO}_{2}$ NPs for photodynamic and photothermal synergistic breast cancer therapy. ${ }^{53}$ Due to PDA's stability at $\mathrm{pH} 7.4$, the premature release of $\mathrm{Ce} 6$ into the blood was avoided. However, PDA was destroyed at $\mathrm{pH} 6.8$ at tumour sites, resulting in the release of $\mathrm{MnO}_{2}$ and Ce6. $\mathrm{MnO}_{2}$ was subsequently decomposed to produce $\mathrm{Mn}^{2+}$ 
(generally used for MRI) and oxygen, which can release PDT by reacting with Ce6. Meanwhile, under NIR light (808 nm), PTT was achieved and the oxygen-burst and release of Ce6 was promoted. Recently, researchers started to focus on the combination of the two above mentioned metal chalcogenides $\left(\mathrm{CuS}\right.$ and $\left.\mathrm{MnO}_{2}\right)$ to obtain various effects. Interestingly, in $\mathrm{CuS} @ \mathrm{MnO}_{2}$ nanocomplexes, $\mathrm{MnO}_{2}$ usually acted as a gatekeeper to prevent the release of loaded drugs in blood circulation and open the "gate" in the acid tumour microenvironment. In Lin's work, ${ }^{72}$ with DOX loaded inside the hollow interior of CuS NPs, multimodal (fluorescence, photothermal and MR) imagingguided controlled release chemotherapy and PTT for liver cancer were achieved. Similarly, Xue et al achieved PA/FL/MR trimodal imaging-guided PDT/PTT synergistic breast cancer therapy with Ce6 loading. ${ }^{54}$ In summary, these types of smart multifunctional nanoplatform have shown great potential for future clinical applications.

\section{Hollow Carbon Nanostructures}

As another promising PTT agent, carbon-based nanomaterials such as carbon nanotubes, ${ }^{73}$ carbon nanohorns ${ }^{74}$ and graphene ${ }^{23}$ have been widely utilized in cancer therapy. As shown in Table 1, more and more hollow carbon nanostructures have been developed for cancer PTT. Similar with gold nanoparticles, carbon nanomaterials generate heat from light via the relaxation of surface electrons. In addition, given the fact that the fluorescence of some carbon nanostructures are prone to be quenched by the mutual effect between different carbon layers or carbon layers with additional components, non-radiative relaxation dominated the de-excitation process of carbon nanostructures, thus most of the energy obtained from light can be converted into heat. ${ }^{75}$ Compared to gold nanoparticles, carbon-based nanoparticles possess tunable photothermal effect in a wide spectral range. ${ }^{76}$ Moreover, hollow carbon nanostructures are also superior in biocompatibility to other nanostructures mentioned, for their abundant surface functional groups and absent metal ions. $^{77}$

However, PTT when used alone is unable to eradicate tumour cells under some conditions. In these case, mesoporous carbon NPs (MCNs) are an ideal option to combine chemotherapy and PTT due to their large pore volume allowing them to serve as drug carriers. In 2017, Li et al developed a new kind of hollow MCN (HMCN) for chemotherapy/PTT for lung cancer and compared its efficacy with that of $\mathrm{MCNs}^{78}$ In that study, DOX was chosen to evaluate the drug loading and release process of the two NPs. With higher drug loading efficiency (up to 76.9\%) and higher photothermal conversion efficiency, HMCNs are more suitable for cancer treatment and show great promise. However, due to their porous surface morphology, premature drug release is an obstacle. In the same year, Wang et al fabricated HMCNs. ${ }^{79}$ To tackle the problem of premature drug release, they used PEI carbon dots as 'doors' for the mesoporous "gate", with disulphide units acting as the 'locks'. When the NPs reached breast cancer cells, GSH overexpressed in cancer cells acted as "keys" to the locks to cleave disulphide bonds, leading to the release of the loaded DOX. Meanwhile, the released $\mathrm{CD}_{\text {PEI }}$ yielded fluorescence, indicating drug release. Then, NIR light irradiation showed that the release rate of DOX was accelerated. In the following year, Fang et al utilized graphene carbon dots to seal the pores, which also functioned as a robust PTT agent in the subsequent synergistic chemo/photothermal cervical cancer treatment. ${ }^{80}$ A triple stimuli-response magnetic hollow porous carbon-based drug delivery system (MHPCNs-SS-PGAFA/DOX) was developed by $\mathrm{Wu}$ et $\mathrm{al}^{81}$. The poly- $\gamma$ glutamic acid-capped shell of hollow NPs included an inner $\mathrm{Fe}_{3} \mathrm{O}_{4}$ layer and a porous carbon outer layer. The authors used silica nanospheres and ferrocene to obtain $\mathrm{SiO}_{2} @ \mathrm{Fe}_{3} \mathrm{O}_{4} @ \mathrm{C}$ via the sacrificial template method. The silica core was then etched using $\mathrm{NH}_{3} \cdot \mathrm{H}_{2} \mathrm{O}$ to form the MHPCNs. MRI-guided therapy was easily performed in mice with cervical cancer and the accumulation of NPs in vivo was monitored by MRI. Moreover, drug release was stimulated by GSH, $\mathrm{pH}$ and NIR light due to the unique properties of the MHPCNs. This guaranteed excellent tumour suppression capacity with negligible side effects in mice.

In addition, Fan et al fabricated nanozymes $(\mathrm{Au} @ \mathrm{HCNs})$ with a yolk-shell structure to achieve catalytic-photothermal colon cancer therapy. ${ }^{82}$ They used $\mathrm{Au}$ as the nanozyme, which functions as a peroxidase and oxidase to regulate reactive oxygen species (ROS). However, unsupported $\mathrm{Au}$ suffered from poor stability, which resulted in a decrease in catalytic efficiency. Thus, carbon shells were used to shelter $\mathrm{Au}$, endowing the nanozymes with high stability in a harsh environment and long-term delivery. Moreover, an ROS burst was observed using irradiation with an 808-nm laser, indicating that the catalytic effect of Au@HCNs could be enhanced by the photothermal effect. The in vivo study verified the authors' 
concept of catalytic-PTT as CT26 tumour growth was successfully inhibited by intravenous administration of $\mathrm{Au}$ @HCNs.

\section{Hollow Silica Nanostructures}

Silica-based NPs have been used as nanocarriers of photosensitisers and drugs in recent years due to their unrivalled biocompatibility (Table 1). As mentioned above, CuS NPs suffer from poor biocompatibility at high concentrations, leading to the development of the two methods described in the previous section. Here, we will introduce a third method: encapsulation by silica shells. In 2007, Li et al developed a dual-imaging-guided multifunctional platform (CuS@mSiO2-TD/ICG) to achieve PDT/PTT synergistic breast cancer therapy. ${ }^{83}$ They coated the NPs with silica shells, which not only enhanced the biocompatibility, but also greatly improved the loading capacity. As a result, ICG, which shared a similar absorption wavelength at 808 $\mathrm{nm}$ with $\mathrm{CuS}$, provided a synergistic photothermal effect with $\mathrm{CuS}$. Moreover, as the temperature increased, the gatekeeper of the nanocarrier, 1-tetradecanol (TD), reached its melting point of $39^{\circ} \mathrm{C}$, followed by a phasechange (solid TD to liquid TD) to allow the controlled release of ICG. ICG then underwent a reaction with oxygen in cancer cells to facilitate photodynamic treatment by yielding ROS. Simultaneously, ICG served as an indicator of the drug distribution in in vivo experiments by yielding fluorescence and PA signals. Early this year, Du et al developed a multimodal imaging-guided interventional therapy for pancreatic cancer. ${ }^{84}$ In this study, Au star endowed the NP, named Gem-perfluorohexane-Au starHMS (GN), with CT and PA imaging capacity. Meanwhile, Gem and perfluorohexane were loaded for chemotherapy and enhancing US imaging, respectively, and the GN was modified with IGF1 to form GN-T for targeted delivery. US, CT and PA imaging then demonstrated that the GN-based nanodrugs could selectively accumulate at the tumour site. Notably, to achieve sustainable release of GN-T, the authors utilised a thermosensitive gel, which solidified after being injected into the tumour site. GN-T was then slowly released with gel erosion. In summary, temperature-controlled PTT and single-dose administrated chemotherapy are promising methods for surgical resection and postoperative chemotherapy to prevent the recurrence of pancreatic cancer.

In 2017, Yu et al developed a novel type of hollow silicon oxide-based $\mathrm{NP}^{85}$ named $\mathrm{H}-\mathrm{SiOx} \mathrm{NPs}$, with a nonstoichiometric ratio $(\mathrm{x}=0.92)$. H-SiOx NPs were synthesised from hollow silica NPs through a magnesiothermic reduction process. This process endowed H-SiOx NPs with a considerable amount of oxygen vacancy, inducing free carriers. With such a high concentration of free carriers, a wide range of absorption from UV to NIR in the second NIR (II) window was ensured. Meanwhile, a high PCE was observed at $1064 \mathrm{~nm}$ (48.6\%), which was higher than that of all siliconbased NPs. Given this property, efficient breast cancer PTT in vivo could be successfully achieved at a low power density $\left(0.6 \mathrm{~W} / \mathrm{cm}^{2}\right)$ under 1064-nm NIR light irradiation. In conclusion, due to their high biocompatibility, silica-based NPs have become one of the safest options for clinical application.

\section{Other Hollow Inorganic Nanostructures Prussian Blue}

Other hollow inorganic nanostructures have also been designed for biological imaging and photothermal-based cancer therapy (Figure 3). ${ }^{86-89}$ Among these, metalorganic frameworks are drawing significant attention due to their attractive properties, such as considerable pore volume and surface area. ${ }^{86}$ Recently, Prussian blue (PB) NPs have been the research focus, since PB was approved for clinical application by the US Food and Drug Administration. That is to say, compared to other nanomaterials mentioned above, Prussian Blue is more likely to be applied to clinical treatment, with the clinical proved biosafety for human body. In 2017, Cai et al developed PB-based NPs (HMNP-PB@Pent@DOX) for trimodal imaging-guided controlled drug release and PTT. ${ }^{90}$ The authors used polystyrene was used as a template, and coated it with a $\mathrm{Fe}_{3} \mathrm{O}_{4}$ shell to function as a $\mathrm{T}_{2}$-weighted MRI contrast agent, followed by coating with PB to form a drug carrier. The drug DOX was loaded/locked with 1-pentadecanol inside the carrier. Under NIR light, PB then converted light into heat, and when the temperature exceeded $42^{\circ} \mathrm{C}$, 1-pentadecanol gradually melted and DOX was released at the liver tumour site. In 2018, Zhou et al fabricated glucose oxidase (GOx)-loaded hollow PB NPs (PHPBNs) for synergetic tumour starvation therapy and low-temperature $\mathrm{PTT}^{21}$ The proliferation of tumour cells requires a considerable amount of ATP, which is mainly generated via anaerobic glycolysis. Thus, to deprive cancer cells of the energy in order to inhibit their growth, GOx was used to consume glucose in tumour cells. Moreover, PHPBNs not only protected GOx from degradation in blood circulation but also produced oxygen, which is essential for the functionalisation of GOx. In addition, PHPBNs ameliorated the tumour resistance 


\section{Other Hollow Inorganic Materials for Cancer PTT}

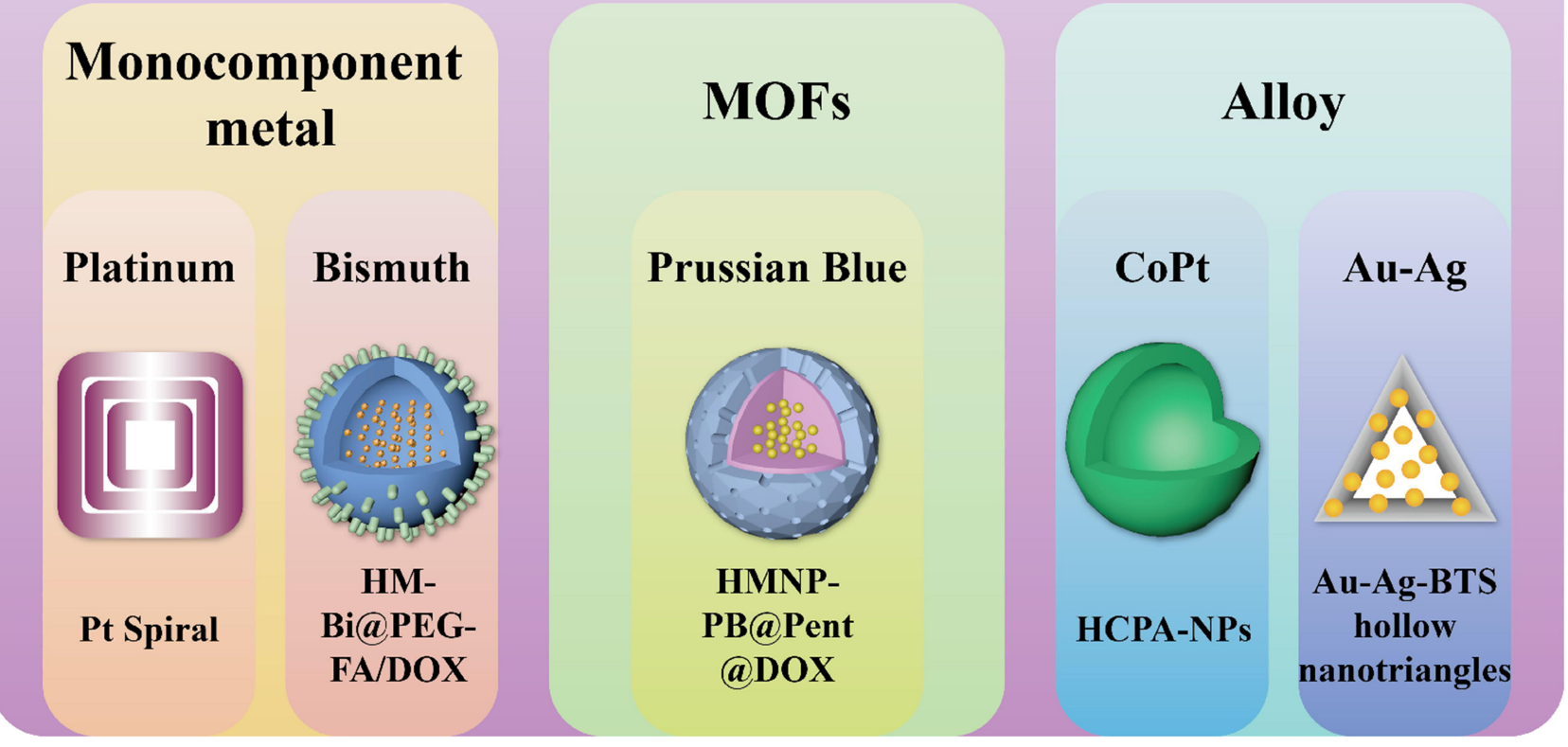

Figure 3 Summary for other hollow inorganic materials for cancer PTT, including monocomponent metals, MOFs and alloys.

problem in a low hyperthermia environment by suppressing the expression of heat shock proteins (HSPs). As HSPs replace heat-denatured proteins and their expression is closely related to the ATP level in cancer cells, ${ }^{91}$ inhibiting ATP production increases the efficiency of lowtemperature PTT. $^{92}$ In an in vivo study, PHPBNs successfully inhibited liver tumour growth by the combination of starvation and mild PTT with almost no side effects in normal tissues.

\section{Monocomponent Metal}

Most recently, PTT using NIR- II window has gained popularity for its superior properties compared to PTT using the NIR-I region, including deeper tissue penetration, lower photon scattering and maximum permissible exposure. ${ }^{93}$ Although the SPR wavelength can be tuned by adjusting the shape and size of the metal nanostructures, few metal NPs for NIR- II PTT have been developed. Notably, because the highorder longitudinal SPR mode of platinum (Pt) NPs is in the NIR zone, it is possible to extend their SPR wavelength to the NIR- II region. ${ }^{94}$ In 2019, Wang et al fabricated hollow Pt nanoframes (Pt Spirals) for CT-guided PTT. ${ }^{95}$ These Pt Spirals were smartly designed with a multilevel structure: first, 1D nanowires were tangentially assembled to produce $2 \mathrm{D}$ nanoshells. Then, 3D nanoframes were constructed by layer-by- layer assembling of 2D nanoshells. This smart design and SPR tuning paved the way for the design of other materials. The researchers proved that the absorption and PCE of the prepared Pt nanoframes were superior to those of solid Pt NPs, with the PCE (52.5\%) representing the highest among all Ptbased NPs. In addition, Pt possesses robust X-ray attenuation, allowing the Pt Spirals to serve as a CT contrast agent. Under CT imaging, the cervix cancer cells were successfully ablated by PTT. Due to their negligible toxicity and low cost, nanomaterials based on bismuth, known as a "green metal", are promising for clinical application. ${ }^{96}$ Three months ago, Huang et al fabricated mono-component hollow bismuth nanoshells (HM-Bi@PEG-FA NSs) for controlled drug delivery and PTT. ${ }^{97}$ When conjugated to folate, bismuth nanoshells could be targeted to tumour sites, where drug release was stimulated by the joint effects of $\mathrm{pH}$ and NIR light irradiation. The therapeutic efficacy of chemo/PTT for lung cancer was then confirmed in in vivo experiments. These nanoshells were found to be an ideal option for clinical application due to their extraordinary biological safety.

\section{Alloy}

In addition to monocomponent metal nanostructures, metal alloys are also a suitable alternative for imaging-guided PTT. Yang et al developed hollow CoPt alloy NPs (HCPA- 
NPs) for PA and MRI-guided liver cancer PTT via a facile, green synthesis route. ${ }^{98}$ Plant polyphenols were used as assistive agents for the formation of HCPA-NPs, of which the sizes could be adjusted by changing the size of the polyphenol. This method could also be used to obtain other hollow alloy NPs and provided a novel green approach for synthesis in other fields such as electronics and catalysis. This year, $\mathrm{Li}$ et al fabricated Au-Ag hollow nanotriangles for gas therapy and PTT. ${ }^{99}$ In their work, the $\mathrm{SO}_{2}$ prodrug benzothiazole sulfinate (BTS) was loaded onto the nanotriangles for effective deep tumour therapy. The $\mathrm{SO}_{2}$ gas was able to diffuse into deep breast tumour tissues despite tumour heterogeneity. After being stimulated in the acidic tumour microenvironment, BTS then released $\mathrm{SO}_{2}$, which, along with PTT, upregulated the expression of Bax in mitochondria and promoted cancer cell apoptosis. Overall, this work offers a promising strategy for deep tumour synergistic therapy.

\section{Photothermal-Based Therapy for Antibacterial Treatment Hollow Gold Nanostructures}

As mentioned earlier, gold nanostructures possess excellent light-to-heat conversion efficiency and can thus be used in antibacterial treatment. Meeker et al developed PDA-coated gold nanocages (AuNCs) for chemo/photothermal synergistic treatment of Staphylococcus aureus infections. ${ }^{100}$ The selectivity of the NPs was achieved by the conjugation of antibodies against staphylococcal protein A. Under NIR light, the consequent temperature rise was sufficient to kill the bacteria and was accompanied by the release of the loaded antibiotic daptomycin from PDA. The results of bactericidal experiments using biofilms showed that with PTT alone, the bacteria were initially eradiated but recovered within 24 hours. In contrast, the synergistic effect of antibiotics and PTT inhibited $S$. aureus growth completely and prevented regrowth. This study provided a robust method for the treatment of biofilm-associated infections to overcome the problem of MDR. In 2018, the authors developed PDA-coated AuNCs with various antibodies and antibiotics to evaluate the versatility of their previous method. ${ }^{101}$ In this study, the authors successfully verified the versatility of the method by inhibiting the growth of the gram-negative pathogen Pseudomonas aeruginosa using a novel combination of antibiotics and antibodies. Further, to tackle the problem of MDR, Zhou et al fabricated gold-silver nanoshells
(AuAgNSs) for wound healing. ${ }^{102}$ As a bactericidal metal ion, $\mathrm{Ag}^{+}$can be released from $\mathrm{Ag}$ NPs under laser irradiation and combined with PTT to exert a synergistic antibacterial effect. However, the photothermal efficiency of $\mathrm{Ag}$ is inferior to that of Au. As such, the authors developed nanoplatforms using both metals. They also conjugated 3.3'-diethylthiatricarbocyanine iodide to track residual bacteria by SERS for 8 weeks.

In recent years, macrophages have been used to achieve targeted drug delivery to tumour sites. This is achieved by coating nanodrugs with the membranes of macrophages. ${ }^{103,104}$ However, the application of this technique to antibacterial drug delivery is rare. Macrophages can be stimulated by various bacteria through different receptors expressed on the macrophage membrane. In one study, Wang et al coated gold-silver nanocages (GSNCs) with macrophage membranes pre-treated with $S$. aureus to form SaM-GSNCs, ${ }^{105}$ and injected the Sa-M-GSNCs subcutaneously and intravenously to treat local and systemic (osteomyelitis) infections. Endowed with prolonged blood circulation time and retention at infected sites, this method paved the way for effective PTT-based antibacterial treatment.

Periodontitis is the main reason for tooth loss ${ }^{106}$ and is generally caused by bacterial infections. Bacterial infections are also implicated in other diseases, such as diabetes and cardiovascular diseases. ${ }^{107}$ To treat bacterial infections, Zhang et al fabricated a tetracycline (TC)-carrying nanoplatform (TC-PCM@GNC-PND) for light-controlled drug release and PTT. ${ }^{108}$ They used phase-change material (PCM) and poly(N-isopropylacrylamide-co-diethylaminoethyl methacrylate) (PND) as two gatekeepers to prevent the premature release of TC. Under NIR laser irradiation, GNC converted light to heat, which then transformed the injectable solid into a gel at the infected site at $36^{\circ} \mathrm{C}$ as a result of the thermosensitive properties of PND. When the temperature rose to $45^{\circ} \mathrm{C}$, PCM melted and released TC. The photothermal effect not only killed the bacteria but also improved the bactericidal efficiency of TC. In in vivo experiments, bone loss was used as an indicator for evaluating tissue destruction stemming from inflammation caused by bacteria. The results showed that the TC-PCM@GNC-PND-treated group experienced minimum bone loss, indicating the excellent therapeutic effect of this method.

\section{Hollow Silica Nanostructures}

Silica nanoshells have been shown to be excellent drug carriers in the chemo/photothermal treatment for cancer. 
However, due to their hollow interior and mesoporous walls, silica nanoshells can also be loaded with antibacterial drugs and combined with PTT to kill bacteria. Wang et al reported kanamycin-encapsulated gold nanorodcovered hollow silica NPs for antibacterial (E. coli BL21) chemo/PTT. ${ }^{109}$ The gold nanorods served as photothermal agents and played the role of "gatekeepers". Apart from the mechanism of gatekeepers mentioned above (phase-change ${ }^{83,108}$ and bond-cleavage), ${ }^{79}$ the authors reported an additional method. Under NIR light irradiation, apart from converting light to heat, the gold nanorods also underwent a shape-changing process before drug release. This synergistic mechanism reduced the drug dosage requirement and shortened the treatment time, providing greater bactericidal efficiency.

Similar to the structure of Sa-M-GSNCs developed by Wang, ${ }^{105}$ silica-coated gold-silver nanocages ( $\mathrm{Au}-$ $\mathrm{Ag} @ \mathrm{SiO} 2 \mathrm{NCs})$ were fabricated by Xu et al ${ }^{110}$. With $\mathrm{Ag}$ ion release, these nanocages achieved NIR-induced PTT both in an S. aureus biofilm model in vitro and in rats with wound infections. Interestingly, $\mathrm{Ji}$ et al produced a novel sandwich-like graphene-mesoporous silica (GS) nanoplatform(AA@GS@HA-MNPs) for targeted drug delivery. In this study, the $\cdot \mathrm{OH}$ prodrug ascorbic acid (AA) was first introduced into the hollow pores, which were subsequently capped with hyaluronic acid-dopamine conjugates (HA-DA) . The targeted release of AA was possible because HA was degraded by hyaluronidase (Hyal), which is overexpressed at bacterial infection sites. ${ }^{111}$ Finally, $\mathrm{Fe}_{3} \mathrm{O}_{4}$ NPs were conjugated to GS to catalyse the $\cdot \mathrm{OH}$-generating effects of AA. The photothermal effect of graphene made synergistic
chemo/PTT possible, dispersing stubborn biofilms and inhibiting bacteria growth. Moreover, the therapy was excellent for treating bacterial infections at tumour sites because cancer cells are the main producers of Hyal. ${ }^{12,113}$ The $\bullet \mathrm{OH}$ produced could kill cancer cells and bacteria simultaneously.

\section{Hollow Metallic Compound Nanostructures}

As mentioned earlier, gold NPs have relatively high toxicity and low biocompatibility. However, in recent years, bismuth-based NPs have become popular due to their unrivalled biocompatibility. ${ }^{56,114,115}$ This year, Qian et al reported a sea-urchin-like $\mathrm{Bi}_{2} \mathrm{~S}_{3}$ hollow nanoplatform (TD/ linalool@Bi2S3) for water sterilization. ${ }^{116}$ In this study, $\mathrm{Bi}_{2} \mathrm{~S}_{3}$ nanoplatforms encapsulated TD combined with the bactericidal agent linalool, the release of which could be controlled with an 808-nm laser to kill the bacteria (E. coli and $S$. aureus) in drinking water along with PTT.

This year, Zhou et al developed dual-functional hollow $\mathrm{AuAgCu} \mathrm{Cu}_{2} \mathrm{O}$ nanospheres with both antibacterial and wound healing effects. ${ }^{117}$ As shown in Figure 4, the former was achieved using a combined therapy including photothermal effects, ROS generation and Ag ion release under laser irradiation to damage the cell walls, proteins and DNA of bacteria (MRSA and ESBL E.coli). The wound healing effects were derived from $\mathrm{Cu}$ ions released from $\mathrm{AuAgCu}_{2}$ $\mathrm{O}$ nanostructures. The $\mathrm{Cu}$ ions were successfully used for promoting re-epithelialisation. The authors then verified the clinical potential of $\mathrm{AuAgCu}_{2} \mathrm{O}$ nanostructures in the treatment of diabetes-related chronic wounds and nonhealing keratitis. Later, the authors investigated ophthalmological

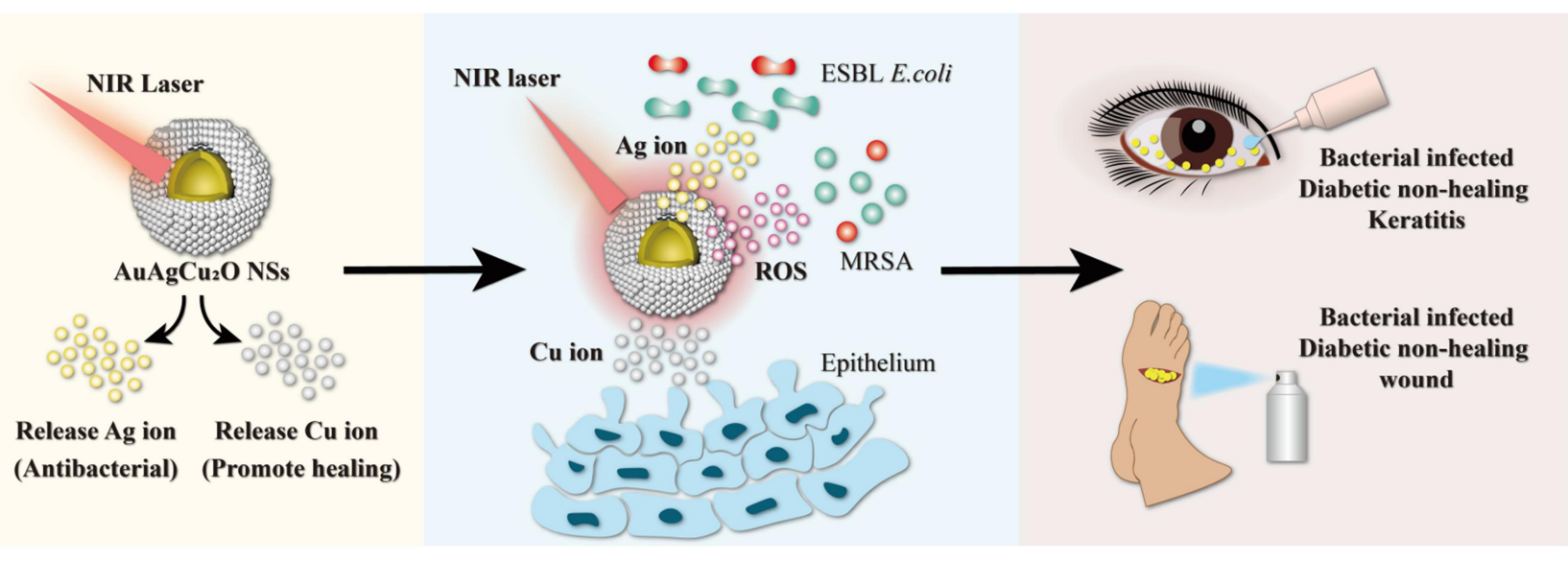

Figure 4 Near-Infrared (NIR)-Activated $\mathrm{AuAgCu}_{2} \mathrm{O}$ Nanoshells for Antibacterial-Resistant Bacterial Killing and Improved Wound Healing. The prepared AuAgCu $\mathrm{O}_{2} \mathrm{O}$ NSs could release copper ions and silver ions under NIR laser irradiation. Consequently, multi-drug-resistant bacteria can be efficiently damaged through a synergistic antibioticphotothermal strategy with silver ions and local high temperature, and the released copper ions could promote the re-epithelialization process, eventually accelerating the recovery of the nonhealing wound and keratitis. Data from Ye et al. ${ }^{117}$ 
diseases, and bromfenac sodium (BS) was conjugated to $\mathrm{AuAgCu} \mathrm{Al}_{2} \mathrm{O} \mathrm{NSs}$ to prevent post-cataract surgery endophthalmitis. ${ }^{118}$ After intraocular injection of $\mathrm{AuAgCu}_{2}$ $\mathrm{O}-\mathrm{BS} \mathrm{NSs}$, the inflammatory symptoms were alleviated and the prognosis was improved due to a mild photothermal effect and the anti-inflammatory drug BS.

\section{Other Hollow Nanostructures}

Some other hollow nanostructures developed for photothermal-based antibacterial therapy have been relatively less reported. An example is hollow lanthanide-doped upconversion NPs (UCNPs), which emit visible light and even UV light under NIR light irradiation and provide relatively deep tissue penetration and low background autofluorescence. ${ }^{119,120}$ Due to these excellent properties, UCNPs are generally used in optical imaging. Given that antibacterial treatment is usually administered in vivo, moderate hyperthermia is crucial because overheating can harm healthy tissues. Thus, integrating a thermometer into a platform is a novel approach for temperature control. In 2017, Guo et al developed $\mathrm{Nd}^{3+} / \mathrm{Yb}^{3+} / \mathrm{Er}^{3+}$ co-doped yolkshell-shaped upconverting thermometer-heater platforms (YS-GOF@Si) for antibacterial PTT. ${ }^{121}$ In this study, the temperature of YS-GOF@Si rose under 808 nm irradiation. This effect was monitored in real time by fluorescence intensity ratio. However, the methods did not perform well in antibacterial experiments, with bacterial (E. coli) viability being $53.1 \%$ after treatment. Later, in 2018, the authors developed another hollow nanothermometer $\left(\mathrm{LuVO}_{4}\right)$ that was also doped with lanthanides. ${ }^{122}$ Furthermore, $\mathrm{Cu}_{2} \mathrm{~S}$ was utilised to modify the oliveshaped NPs, which could absorb both incident light and NIR light from a luminescent core, providing excellent thermal sensitivity based on spectrally pure green emission. In addition, the nanothermometers prepared in this study possessed much higher bactericidal (E. coli and S. aureus) efficiency ( 95\%) than YS-GOF@Si. Overall, Guo et al provided a promising method for temperature-monitored, minimally invasive PTT.

In the cancer treatment section, we introduced Prussian blue as a member of the MOF family. Here, we introduce another member: HKUST-1. This year, Yu et al used HKUST-1 frameworks to encapsulate CuS NPs by converting trace amounts of $\mathrm{Cu}$ ions into $\mathrm{CuS}$ NPs. Under NIR light, the HKUST-1-supported CuS NPs could generate ROS and kill bacteria (E. coli and $S$. aureus) in combination with PTT. ${ }^{123}$ Due to the protection provided by the framework, the aggregation and direct tissue contact of CuS NPs was avoided. By applying multimodal antibacterial therapy, including PTT, PDT and $\mathrm{Cu}$ ion release, a bactericidal effect of over 99\% was achieved, demonstrating great potential for biomedical applications.

\section{Photothermal-Based Therapy for the Treatment of Other Diseases Alzheimer's Disease}

As a common neurodegenerative disease, $\mathrm{AD}$ is usually seen in patients with dementia ${ }^{124}$ and is considered a worldwide health problem. ${ }^{125}$ The extracellular accumulation of amyloid plaques is one of the hallmarks of $\mathrm{AD}$. These plaques are mainly composed of amyloid- $\beta$ peptides $(A \beta) .{ }^{126}$ Given this fact, a promising strategy for treating $\mathrm{AD}$ involves preventing $A \beta$ aggregation and destroying the already-formed $A \beta$ fibrils. To date, various therapies for inhibiting $A \beta$ aggregation have been investigated. ${ }^{127-129}$ However, these methods often provide insufficient inhibition of aggregation and suffer from a poor disaggregating capacity. Therefore, localised PTT may be a robust tool and an ideal hyperthermia therapy for $\mathrm{AD}$ treatment, with minimal side effects to surrounding tissues.

In 2017, Ruff et al developed hollow Au NPs (HAuNS) conjugated with CLPFFD peptides for selectively binding $\mathrm{A} \beta$ structures. ${ }^{130}$ They fabricated CLPFFD-PEG-HAuNS via two approaches, first by binding the CLPFFD peptides directly to the HAuNS and second by binding the peptides indirectly to a PEG ligand shell. The authors used in vitro blood-brain barrier (BBB) model to prove that the impedance of BBB passage caused by the negative charge on the peptide was countered by coupling peptides to the PEG ligand. The $A \beta$ aggregation-inhibiting effect of CLPFFD peptide-modified HAuNS was demonstrated experimentally in a later work ${ }^{131}$ and in in vivo work performed previously. ${ }^{132,133}$ These studies showed that HAuNS has potential for application in AD treatment.

The hyperphosphorylation of tau protein is also a main culprit in $\mathrm{AD}$, contributing to the aggregation of the protein followed by the production of ROS. ${ }^{134}$ Abnormal production of ROS in the brain results in inflammatory reactions, which can impair neuron function and cause neuronal apoptosis, consequently influencing the basic functions of the brain such as learning and memory. In case of $\mathrm{AD}$, repairing the impaired neurons and preventing tau protein hyperphosphorylation represent a promising treatment for AD. This year, Zhou et al fabricated nerve growth factor (NGF)-loaded hollow ruthenium (NGF- 
PCM $@$ Ru) nanoflowers for AD treatment. ${ }^{135}$ As a member of the neurotrophin family, NGF could serve as an inhibitor of tau hyperphosphorylation. However, the very low rate of $\mathrm{BBB}$ passage and short blood circulation time limited its biological application. As shown in Figure 5, Ru NPs were utilised as carriers for NGF. Under NIR laser irradiation, BBB permeability was enhanced, helping the nanocarriers to enrich in brain tissue. Simultaneously, as the temperature rose, NGF was released via a phase-change process of PCM. Using the water maze test and nesting experiments, the authors proved the validity of NGF-PCM@Ru in rescuing memory loss in mice with AD.

\section{Obesity}

In modern society, obesity is a fast-growing disease that can cause serious health impairments. ${ }^{136}$ In children in particular, the obesity ratio dramatically increased to $47 \%$ in the $30+$ years from 1980 to $2013 .{ }^{137}$ In addition, obesity is implicated in many chronic diseases, including

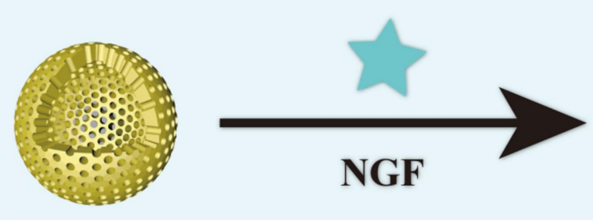

\section{RuNPs}
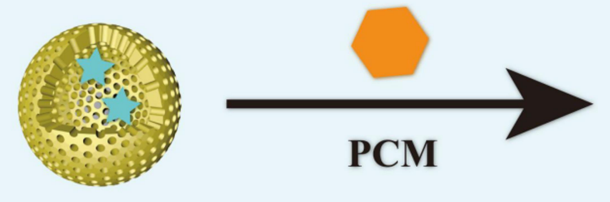

NGF-PCM@RuNPs

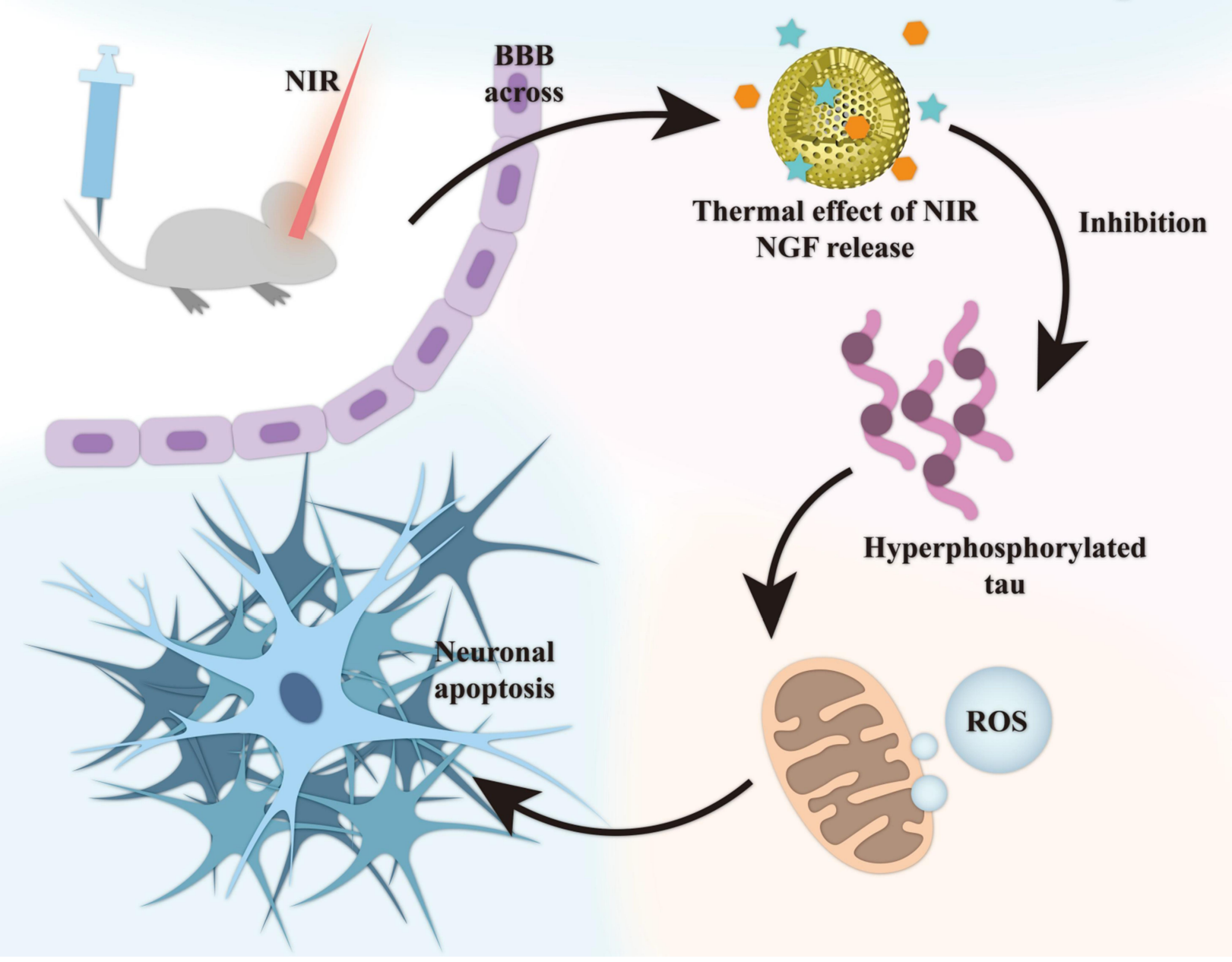

Figure 5 NGF-PCM@Ru NPs were used to cross BBB under NIR irradiation, and then PCM triggered the release of NGF in response to thermal effects, thereby achieving reduction of ROS production and mitigation of neuronal damage by inhibiting tau hyperphosphorylation. Data from Zhou et al. ${ }^{135}$ 
ischemic stroke, $^{138}$ type-2 diabetes ${ }^{139}$ and fatty liver disease. ${ }^{140}$ At present, liposuction and anti-obesity drugs are the two major treatment methods to combat obesity. However, discomfort and pain caused by liposuction and side effects stemming from drug abuse are the major drawbacks restricting their clinical application.

Recently, a novel obesity treatment that utilised NPs was developed. ${ }^{141}$ The therapeutic strategy in most of these treatments was photothermal lipolysis. Han and Kim developed polypyrrole (PPy)-covered hollow gold nanoshells (HAuNS@PPy) for adipocyte ablation ${ }^{142}$ and performed ex vivo experiments to evaluate their therapeutic efficiency. They found that subcutaneous adipose tissue was degraded, along with apoptosis of adipocytes. However, this method non-selectively ablated both adipose tissues and normal tissues, limiting its clinical application. To address this limitation, Lee et al developed HAHAuNS-ATP for targeted transdermal delivery of the nanoshells. ${ }^{143}$ In this study, hyaluronate was conjugated to HAuNS, endowing the nanoshells with transdermal ability, and the targeting capacity of the nanoshells was derived from the ATP sequence. The nanodrugs penetrated the epidermis and targeted adipocytes. Under laser irradiation, adipocytes were ablated by the photothermal effect, which could be visualised using PA imaging. The results showed that $20 \%$ of the initial lipid was eliminated, thus demonstrating a potential novel non-invasive therapy for obesity.

\section{Endometriosis}

Endometriosis is a common oestrogen-dependent gynaecological disease, which is defined as the growth of endometrial cells outside the uterine cavity. ${ }^{144}$ Endometriosis is implicated in many health problems, including dysmenorrhea, dyspareunia, pelvic pain and infertility. ${ }^{145,146}$ The disease affects $10 \%$ of women of reproductive age. In patients with endometriosis, the endometrial cells exhibit a decreased rate of apoptosis and an increased rate of proliferation. The environment in the ectopic endometrial tissue can prompt the implantation of endometrial cells and help them escape immune clearance. ${ }^{147,148}$ At present, there is no cure for this disorder, and the most frequently used treatment strategy is surgical, which is associated with a high recurrence rate $(>50 \%)$ due to from the presence of endometriotic residues after surgical excision. ${ }^{149}$ Given that endometriosis is similar to solid cancer in many aspects and the diseases are usually concomitant, ${ }^{150} \mathrm{NP}$ - based PTT could be an ideal therapeutic strategy for endometriosis.

In 2017, Guo et al developed targeted HAuNS for photothermal-based endometriosis therapy. ${ }^{151}$ Neovascularisation, a common feature of both cancer and endometriosis, is closely associated with the overexpression of Eph receptors. To achieve the targeting effect, HAuNS was conjugated with TNYL peptides, which possess remarkable binding efficiency to EphB4 receptors. In in vivo experiments, under NIR light irradiation, TNYL-HAuNS remarkably inhibited lesion growth by photothermal ablation, with negligible damage to normal tissues. However, patients whose uterus is in a congestive state (menses) cannot be treated with this method since EphB4 is also highly expressed in the uterus.

\section{Conclusion and Perspectives}

In this review, we discussed the recent biomedical applications of hollow inorganic nanomaterials in PTT for various diseases. PTT, along with the advancements in nanotechnology, has been widely investigated by scientists owing to the high therapeutic efficiency of PTT and the properties of hollow nanoplatforms, which can provide an ideal method for combining different therapies and enhance the treatment efficiency. Specifically, the hollow structures can significantly boost the loading capacity of nanostructures, allowing them to serve as perfect carriers for imaging contrast agents and therapeutic drugs. With the help of PTT, controlled drug release can be realised at laserirradiated sites. Given these properties, many theranostic nanomedicines (based on gold, metal chalcogenides, carbon, silica, etc.) have been developed to treat intractable health problems. First, we introduced the application of hollow inorganic nanomedicines to PTT-based cancer treatment. By loading various drugs and agents on nanostructures, a combination therapy consisting of PTT and other therapies, such as chemotherapy (DOX-loaded) and photodynamic therapy (Ce6- or ICG-loaded) can be achieved to improve therapeutic efficacy and prevent cancer recurrence. Moreover, loading of contrast agents can allow precise monitoring using a combination of MR, PA, fluorescence and US imaging. Second, we discussed a promising strategy for combating infections caused by drug-resistant bacteria. The synergistic effects of antibiotics and hyperthermia can effectively inhibit bacterial growth, preventing "superbug" drug resistance. Third, we mentioned the application of hollow inorganic nanomedicines for the treatment of other diseases, including $\mathrm{AD}$, obesity and endometriosis. As in the cases of cancer and 
bacterial infections, the mechanism underlying the treatment of obesity and endometriosis with hollow inorganic nanomedicine was the ablation of adipocytes and endometrial cells. However, apart from the ablative effect and controlled drug release, NIR light-induced hyperthermia had a novel usage in AD treatment, which was to increase the BBB permeability of nanodrugs. These studies suggest a novel approach for the development of drugs for brain diseases. Drugs for other diseases may also benefit from this enhanced permeability of biological barriers.

All of these achievements suggest the promise of applying hollow inorganic nanomedicines for treatment in clinical patients. However, to date, according to the data from ClinicalTrials.gov, only 19 photothermal therapies have been registered for clinical trials, of which only two nanomedicines have completed clinical trials. Different from traditional energy-based ablation therapies including high energy laser and focused ultrasound, nanoparticle-based tissue ablation can achieve high specificity towards solid tumor, thereby contributing to relative negligible side-effects to normal tissue. One of the two nanophotothermal therapies named NANOM-FIM (Identifier:NCT01270139) was successfully carried out. In this study, silica-gold nanoparticles were delivered by a bioengineered stem cell patch, with the help of minimally invasive cardiac surgery (MICS CABG), significant regression of coronary atherosclerosis was achieved through plasmonic photothermal therapy. ${ }^{152}$ Moreover, the long-term outcome showed that NANOMFIM was superior to stent XIENCE $\mathrm{V}$ in safety and mortality. ${ }^{153}$ The clinical trial of the other nanomedicine was gold nanoshells, named Auroshell ${ }^{\circledR}$ (Identifier: NCT00848042) they were developed to treat head and neck and prostate cancers. Although their preclinical experiments demonstrated that Auroshell ${ }^{\circledR}$ showed low toxicity to Beagle dogs, ${ }^{154}$ some patients still had serious side-effects during the clinical trial, where 11 people were involved and only 5 people completed the trial. We believe that the main clinical limitation of hollow inorganic PTAs is their safety and toxicity. However, these drawbacks can be overcome to some extent. In this review, we described three methods for alleviating chronic toxicity and improving safety, including using biodegradable materials, reducing the dose of NPs and encapsulating NPs in biocompatible materials such as silica. More experiments using cell or animal models should be performed to ensure the safety of nanomedicines, following these suggested approaches. Moreover, insufficient PCE and photothermal stability are further obstacles in the clinical application of hollow inorganic nanomedicines. Thus, scientists should focus on improving the photothermal stability and PCE of nanomedicines to ensure sufficient photothermal effects at lower doses. Notably, although robust photothermal ablation could efficiently eliminate the target cells, the surrounding normal tissues are also likely to undergo apoptosis. Thus, the PTT strategy for each disease should be designed to be disease-specific. Specifically, higher temperatures can be used to kill cancer cells, whereas only mild hyperthermia should be used at some bacterial infection sites, such as eyes. In addition, the clinical application of many photothermal therapies are facing challenges such as limited light penetration depth. For most NIR-I PTAs, light penetration depth is confined to several millimeters due to the tissue scattering; however, as mentioned, by using NIR-II PTAs the penetration depth would be deeper, along with higher maximum permissible exposure (MPE), the clinical application of noninvasive deep tissue PTT would be possible. At present, for some diseases including macular diseases, acne vulgaris, oral cancer, and gastric cancer, NIR laser can be easily delivered to the superficial lesion via direct irradiation or gastroscope. While, for some diseases whose lesions are deeply buried in abdomen, such as pancreatic cancer (PC), NIR light cannot be delivered to the lesion directly. Recently, a novel method named interventional PTT (IPTT) was employed by Tian's group to ablate PC deep in the abdomen. ${ }^{155}$ In IPTT, an NIR optical fiber runs through an 18-gauge $(\mathrm{G})$ percutaneous transhepatic cholangiography (PTC) needle to form the IPTT device. By using this device, NIR light can be easily delivered to the deep-buried tumor site; moreover, precise PTT can also be achieved, thereby reducing the ablative effect on normal tissues.

At present, many researchers are focusing on combating cancer, for which a considerable number of hollow inorganic nanoscale PTAs have been developed compared to those for other diseases. Here, we described the potential of these materials in treating other health problems, including bacterial infections, $\mathrm{AD}$, obesity and endometriosis. We hope that this review can arouse the interest of researchers in applying hollow inorganic nanoscale PTAs to treat these conditions, and that in the future, more diseases would be successfully treated using hollow inorganic nanomaterial-based PTT.

\section{Acknowledgment}

This work was supported by the Ministry of Science and Technology of China (NO.2017ZX09101001-005-003), the National Natural Science Foundation of China (NO.81972892 and NO.81673364) and the Applied 
Technology Research and the Development Project of the Inner Mongolia Autonomous Region (2019GG035).

\section{Disclosure}

The authors report no conflicts of interest related to this work.

\section{References}

1. Siegel RL, Miller KD, Jemal A. Cancer statistics, 2020. $C A$ : Cancer J Clin. 2020;70(1):7-30. doi:10.3322/caac.21590

2. Singhal S, Nie S, Wang MD. Nanotechnology applications in surgical oncology. Annu Rev Med. 2010;61:359-373. doi:10.11 46/annurev.med.60.052907.094936

3. Chi A, Liao Z, Nguyen NP, Xu J, Stea B, Komaki R. Systemic review of the patterns of failure following stereotactic body radiation therapy in early-stage non-small-cell lung cancer: clinical implications. Radiother Oncol. 2010;94(1):1-11. doi:10. 1016/j.radonc.2009.12.008

4. Senapati S, Mahanta AK, Kumar S, Maiti P. Controlled drug delivery vehicles for cancer treatment and their performance. Signal Transduct Target Ther. 2018;3(7). doi:10.1038/s41392017-0004-3

5. Xiao Q, Zheng X, Bu W, et al. A core/satellite multifunctional nanotheranostic for in vivo imaging and tumor eradication by radiation/photothermal synergistic therapy. $J$ Am Chem Soc. 2013;135(35):13041-13048. doi:10.1021/ja404985w

6. Ray PC, Khan SA, Singh AK, Senapati D, Fan Z. Nanomaterials for targeted detection and photothermal killing of bacteria. Chem Soc Rev. 2012;41(8):3193-3209. doi:10.1039/c2cs15340h

7. Vikesland PJ, Wigginton KR. Nanomaterial enabled biosensors for pathogen monitoring - a review. Environ Sci Technol. 2010;44 (10):3656-3669. doi:10.1021/es903704z

8. Wright GD, McCarthy MI. Molecular mechanisms of antibiotic resistance. Chem Commun. 2011;47(14):4055-4061. doi:10.1039/ $\mathrm{c} 0 \mathrm{cc} 05111 \mathrm{j}$

9. Fair RJ, Tor Y. Antibiotics and bacterial resistance in the $21 \mathrm{st}$ century. Perspect Medicin Chem. 2014;6:25-64. doi:10.4137/ PMC.S14459

10. Zou L, Wang H, He B, et al. Current approaches of photothermal therapy in treating cancer metastasis with nanotherapeutics. Theranostics. 2016;6(6):762-772. doi:10.7150/thno.14988

11. Shanmugam V, Selvakumar $S$, Yeh C. Near-infrared light-responsive nanomaterials in cancer therapeutics. Chem Soc Rev. 2014;43(17):6254-6287. doi:10.1039/C4CS00011K

12. Huang XH, El-Sayed IH, Qian W, El-Sayed MA. Cancer cell imaging and photothermal therapy in the near-infrared region by using gold nanorods. J Am Chem Soc. 2006;128(6):2115-2120. doi: $10.1021 / \mathrm{ja} 057254 \mathrm{a}$

13. Wang J, Wu X, Shen $\mathrm{P}$, et al. Applications of inorganic nanomaterials in photothermal therapy based on combinational cancer treatment. Int J Nanomedicine. 2020;15:1903-1914. doi:10.21 47/IJN.S239751

14. Xu JW, Yao K, Xu ZK. Nanomaterials with a photothermal effect for antibacterial activities: an overview. Nanoscale. 2019;11 (18):8680-8691. doi:10.1039/C9NR01833F

15. Jain PK, Huang X, El-Sayed IH, El-Sayed MA. Noble metals on the nanoscale: optical and photothermal properties and some applications in imaging, sensing, biology, and medicine. Accounts Chem Res. 2008;41(12):1578-1586. doi:10.1021/ ar7002804

16. Chen J, Ning C, Zhou Z, et al. Nanomaterials as photothermal therapeutic agents. Prog Mater Sci. 2019;99:1-26. doi:10.1016/j. pmatsci.2018.07.005
17. Liu Y, Bhattarai P, Dai Z, Chen X. Photothermal therapy and photoacoustic imaging via nanotheranostics in fighting cancer. Chem Soc Rev. 2019;48(7):2053-2108.

18. Zhou G, Xiao H, Li X, et al. Gold nanocage decorated $\mathrm{pH}$-sensitive micelle for highly effective photothermo-chemotherapy and photoacoustic imaging. Acta Biomater. 2017;64:223-236. doi:10.1016/j.actbio.2017.10.018

19. You Q, Sun Q, Yu M, et al. BSA-bioinspired gadolinium hybrid-functionalized hollow gold nanoshells for NIRF/PA/CT/ MR quadmodal diagnostic imaging-guided photothermal/photodynamic cancer therapy. ACS Appl Mater Interfaces. 2017;9 (46):40017-40030. doi:10.1021/acsami.7b11926

20. Guo L, Yan DD, Yang D, et al. Combinatorial photothermal and immuno cancer therapy using chitosan-coated hollow copper sulfide nanoparticles. Acs Nano Nano. 2014;8(6):5670-5681. doi:10.1021/nn5002112

21. Zhou J, Li M, Hou Y, et al. Engineering of a nanosized biocatalyst for combined tumor starvation and low-temperature photothermal therapy. Acs Nano Nano. 2018;12(3):2858-2872. doi:10.1021/ acsnano.8b00309

22. Kennedy LC, Bickford LR, Lewinski NA, et al. A new era for cancer treatment: gold-nanoparticle-mediated thermal therapies. Small. 2011;7(2):169-183. doi:10.1002/smll.201000134

23. Yang K, Zhang S, Zhang G, Sun X, Lee S, Liu Z. Graphene in mice: ultrahigh in vivo tumor uptake and efficient photothermal therapy. Nano Lett. 2010;10(9):3318-3323. doi:10.1021/nl100 996u

24. Cheng Y, Hu J, Qin S, Zhang A, Zhang X. Recent advances in functional mesoporous silica-based nanoplatforms for combinational photo-chemotherapy of cancer. Biomaterials. 2020;23 2:119738. doi:10.1016/j.biomaterials.2019.119738

25. Huang X, Zhang W, Guan G, Song G, Zou R, Hu J. Design and functionalization of the NIR-responsive photothermal semiconductor nanomaterials for cancer theranostics. Accounts Chem Res. 2017;50(10):2529-2538. doi:10.1021/acs.accounts.7b00294

26. Xu L, Cheng L, Wang C, Peng R, Liu Z. Conjugated polymers for photothermal therapy of cancer. Polym Chem-UK. 2014;5 (5):1573-1580. doi:10.1039/C3PY01196H

27. Lovell JF, Jin CS, Huynh E, et al. Porphysome nanovesicles generated by porphyrin bilayers for use as multimodal biophotonic contrast agents. Nat Mater. 2011;10(4):324-332. doi:10.10 $38 /$ nmat2986

28. Hartland GV. Coherent excitation of vibrational modes in metallic nanoparticles. Annu Rev Phys Chem. 2006;57(1):403-430. doi:10.1146/annurev.physchem.57.032905.104533

29. Wang J, Li N. Functional hollow nanostructures for imaging and phototherapy of tumors. J Mater Chem B. 2017;5(43):8430-8445. doi:10.1039/C7TB02381B

30. Hao E, Li S, Bailey RC, Zou S, Schatz GC, Hupp JT. Optical properties of metal nanoshells. J Phys Chem B. 2004;108 (4):1224-1229. doi:10.1021/jp036301n

31. Ren Q, Bai L, Zhang X, et al. Preparation, modification, and application of hollow gold nanospheres. J Nanomater. 2015; 2015:534070. doi: $10.1155 / 2015 / 534070$

32. You J, Zhang G, Li C. Exceptionally high payload of doxorubicin in hollow gold nanospheres for near-infrared light-triggered drug release. Acs Nano Nano. 2010;4(2):1033-1041. doi:10.1021/ nn901181c

33. Kwizera EA, Chaffin E, Shen X, et al. Size- and shape-controlled synthesis and properties of magnetic-plasmonic core-shell nanoparticles. $J$ Phys Chem C Nanomater Interfaces. 2016;120 (19):10530-10546. doi:10.1021/acs.jpcc.6b00875

34. Bao Z, Liu X, Liu Y, Liu H, Zhao K. Near-infrared light-responsive inorganic nanomaterials for photothermal therapy. Asian J Pharm Sci. 2016;11(3):349-364. doi:10.1016/j. ajps.2015.11.123 
35. Gao D, Guo X, Zhang X, et al. Multifunctional phototheranostic nanomedicine for cancer imaging and treatment. Materials Today Bio. 2020;5:100035. doi:10.1016/j.mtbio.2019.100035

36. Yu Z, Chan WK, Zhang Y, Tan T. Near-infrared-II activated inorganic photothermal nanomedicines. Biomaterials. 2020;12 0459. doi:10.1016/j.biomaterials.2020.120459

37. Austin LA, Mackey MA, Dreaden EC, El-Sayed MA. The optical, photothermal, and facile surface chemical properties of gold and silver nanoparticles in biodiagnostics, therapy, and drug delivery. Arch Toxicol. 2014;88(7):1391-1417.

38. Jain PK, Huang X, El-Sayed IH, El-Sayad MA. Review of some interesting surface plasmon resonance-enhanced properties of noble metal nanoparticles and their applications to biosystems. Plasmonics. 2007;2(3):107-118. doi:10.1007/s11468-007-9031-1

39. Wang R, Deng J, He D, et al. PEGylated hollow gold nanoparticles for combined X-ray radiation and photothermal therapy in vitro and enhanced CT imaging in vivo. Nanomedicine-Uk. 2019;16:195-205. doi:10.1016/j.nano.2018.12.005

40. Li Y, He D, Tu J, et al. The comparative effect of wrapping solid gold nanoparticles and hollow gold nanoparticles with doxorubicin-loaded thermosensitive liposomes for cancer thermo-chemotherapy. Nanoscale. 2018;10(18):8628-8641. doi:10. 1039/C7NR09083H

41. Cai K, Zhang W, Zhang J, Li H, Han H, Zhai T. Design of gold hollow nanorods with controllable aspect ratio for multimodal imaging and combined chemo-photothermal therapy in the second near-infrared window. ACS Appl Mater Interfaces. 2018;10(43):36703-36710. doi:10.1021/acsami.8b12758

42. Zhan C, Huang Y, Lin C, Huang S, Zeng F, Wu S. A gold nanocage/cluster hybrid structure for whole-body multispectral optoacoustic tomography imaging, EGFR inhibitor delivery, and photothermal therapy. Small. 2019;15:190030933. doi:10.1002/ smll.201900309

43. Feng Y, Cheng Y, Chang Y, et al. Time-staggered delivery of erlotinib and doxorubicin by gold nanocages with two smart polymers for reprogrammable release and synergistic with photothermal therapy. Biomaterials. 2019;217:119327. doi:10.1016/j. biomaterials.2019.119327

44. Huang S, Liu Y, Xu X, et al. Triple therapy of hepatocellular carcinoma with microRNA-122 and doxorubicin co-loaded functionalized gold nanocages. $J$ Mater Chem B. 2018;6 (15):2217-2229. doi:10.1039/C8TB00224J

45. Hirsch LR, Stafford RJ, Bankson JA, et al. Nanoshell-mediated near-infrared thermal therapy of tumors under magnetic resonance guidance. Proc Natl Acad Sci USA. 2003;100(23):13 549-13554. doi:10.1073/pnas.2232479100

46. Wang J, Wheeler D, Zhang JZ, Achilefu S, Kang KA. NIR fluorophore-hollow gold nanosphere complex for cancer enzymetriggered detection and hyperthermia. In: Welch WJ, Palm F, Bruley DF, Harrison DK, editors. Advances in Experimental Medicine and Biology. Springer; Vol. 765. 2013:323-328.

47. Chen J, McLellan JM, Siekkinen A, Xiong Y, Li Z, Xia Y. Facile synthesis of gold-silver nanocages with controllable pores on the surface. J Am Chem Soc. 2006;128(46):14776-14777. doi:10.10 21/ja066023g

48. Yavuz MS, Cheng Y, Chen J, et al. Gold nanocages covered by smart polymers for controlled release with near-infrared light. Nat Mater. 2009;8(12):935-939. doi:10.1038/nmat2564

49. Chen JY, Wiley B, Li ZY, et al. Gold nanocages: engineering their structure for biomedical applications. Adv Mater. 2005;17 (18):2255-2261. doi:10.1002/adma.200500833

50. Zhao Y, Pang B, Detering L, et al. Melanocortin I receptor targeted imaging of melanoma with gold nanocages and positron emission tomography. Mol Imaging. 2018;17:153601211877582. doi:10.1177/1536012118775827
51. Wang Y, An L, Lin J, Tian Q, Yang S. A hollow Cu9S8 theranostic nanoplatform based on a combination of increased active sites and photothermal performance in enhanced chemodynamic therapy. Chem Eng J. 2020;385:123925. doi:10.1016/j.cej.20 19.123925

52. Wang D, Dong H, Li M, et al. Erythrocyte-cancer hybrid membrane camouflaged hollow copper sulfide nanoparticles for prolonged circulation life and homotypic-targeting photothermal/ chemotherapy of melanoma. Acs Nano Nano. 2018;12(6): 5241-5252. doi:10.1021/acsnano.7b08355

53. Zeng $\mathrm{W}$, Zhang $\mathrm{H}$, Deng $\mathrm{Y}$, et al. Dual-response oxygen-generating $\mathrm{MnO} 2$ nanoparticles with polydopamine modification for combined photothermal-photodynamic therapy. Chem Eng J. 2020;389:124494. doi:10.1016/j.cej.2020.124494

54. Li Q, Ren J, Chen Q, et al. A HMCuS@MnO(2)nanocomplex responsive to multiple tumor environmental clues for photoacoustic/fluorescence/magnetic resonance trimodal imaging-guided and enhanced photothermal/photodynamic therapy. Nanoscale. 2020;12(23):12508-12521. doi:10.1039/D0NR01547D

55. Zhang C, Li D, Pei P, et al. Rod-based urchin-like hollow microspheres of Bi2S3: facile synthesis, photo-controlled drug release for photoacoustic imaging and chemo-photothermal therapy of tumor ablation. Biomaterials. 2020;237:119835. doi:10.1016/j. biomaterials.2020.119835

56. Song Y, Wang Y, Zhu Y, et al. Biomodal tumor-targeted and redox-responsive Bi2Se3 hollow nanocubes for MSOT/CT imaging guided synergistic low-temperature photothermal radiotherapy. Adv Healthc Mater. 2019;8(16):1900250. doi:10.10 02/adhm.201900250

57. Sun S, Li P, Liang S, Yang Z. Diversified copper sulfide (Cu2-xS) micro-/nanostructures: a comprehensive review on synthesis, modifications and applications. Nanoscale. 2017;9(32):11357-11404.

58. Dong K, Liu Z, Li Z, Ren J, Qu X. Hydrophobic anticancer drug delivery by a $980 \mathrm{~nm}$ laser-driven photothermal vehicle for efficient synergistic therapy of cancer cells in vivo. Adv Mater. 2013;25(32):4452-4458. doi:10.1002/adma.201301232

59. Guo L, Panderi I, Yan DD, et al. A comparative study of hollow copper sulfide nanoparticles and hollow gold nanospheres on degradability and toxicity. Acs Nano Nano. 2013;7 (10):8780-8793. doi:10.1021/nn403202w

60. Qiu Y, Lin M, Chen G, et al. Photodegradable CuS SERS probes for Intraoperative residual tumor detection, ablation, and self-clearance. ACS Appl Mater Inter. 2019;11(26):2343 6-23444. doi:10.1021/acsami.9b00469

61. Zhang H, Chen Y, Cai Y, et al. Paramagnetic CuS hollow nanoflowers for T-2-FLAIR magnetic resonance imaging-guided thermochemotherapy of cancer. Biomater Sci-UK. 2019;7 (1):409-418. doi:10.1039/C8BM01412D

62. Brady DC, Crowe MS, Turski ML, et al. Copper is required for oncogenic BRAF signalling and tumorigenesis. Nature. 2014;509 (7501):492. doi:10.1038/nature13180

63. Martin F, Linden T, Katschinski DM, et al. Copper-dependent activation of hypoxia-inducible factor (HIF)-1: implications for ceruloplasmin regulation. Blood. 2005;105(12):4613-4619. doi:10.1182/blood-2004-10-3980

64. Jain S, Cohen J, Ward MM, et al. Tetrathiomolybdate-associated copper depletion decreases circulating endothelial progenitor cells in women with breast cancer at high risk of relapse. Ann Oncol. 2013;24(6):1491-1498. doi:10.1093/annonc/mds654

65. Guan G, Wang X, Huang X, et al. Porous cobalt sulfide hollow nanospheres with tunable optical property for magnetic resonance imaging-guided photothermal therapy. Nanoscale. 2018;10 (29):14190-14200. doi:10.1039/C8NR01926F

66. Ding B, Zheng P, Ma P, Lin J. Manganese oxide nanomaterials: synthesis, properties, and theranostic applications. Adv Mater. 2020;32:190582310. doi:10.1002/adma.201905823 
67. An K, Kwon SG, Park M, et al. Synthesis of uniform hollow oxide nanoparticles through nanoscale acid etching. Nano Lett. 2008;8(12):4252-4258. doi:10.1021/n18019467

68. Shin J, Anisur RM, Ko MK, Im GH, Lee JH, Lee IS. Hollow manganese oxide nanoparticles as multifunctional agents for magnetic resonance imaging and drug delivery. Angew Chem Int Ed. 2009;48(2):321-324. doi:10.1002/anie.200802323

69. Chen Y, Yin Q, Ji X, et al. Manganese oxide-based multifunctionalized mesoporous silica nanoparticles for $\mathrm{pH}$-responsive MRI, ultrasonography and circumvention of MDR in cancer cells. Biomaterials. 2012;33(29):7126-7137. doi:10.1016/j.biomaterials.2012.06.059

70. Wang L, Guan S, Weng Y, et al. Highly efficient vacancy-driven photothermal therapy mediated by ultrathin $\mathrm{MnO} 2$ nanosheets. ACS Appl Mater Inter. 2019;11(6):6267-6275. doi:10.1021/ acsami.8b20639

71. Li X, Feng X, Sun C, Liu Y, Zhao Q, Wang S. Mesoporous carbon-manganese nanocomposite for multiple imaging guided oxygen-elevated synergetic therapy. $J$ Control Release. 2020;319:104-118. doi:10.1016/j.jconrel.2019.12.042

72. Lin X, Fang Y, Tao Z, et al. Tumor-microenvironment-induced all-in-one nanoplatform for multimodal imaging-guided chemical and photothermal therapy of cancer. ACS Appl Mater Inter. 2019;11(28):25043-25053. doi:10.1021/acsami.9b07643

73. Kam N, O’Connell M, Wisdom JA, Dai HJ. Carbon nanotubes as multifunctional biological transporters and near-infrared agents for selective cancer cell destruction. Proc Natl Acad Sci USA. 2005;102(33):11600-11605. doi:10.1073/pnas.0502680102

74. Chen D, Wang C, Jiang F, Liu Z, Shu C, Wan L. In vitro and in vivo photothermally enhanced chemotherapy by single-walled carbon nanohorns as a drug delivery system. J Mater Chem B. 2014;2(29):4726-4732. doi:10.1039/C4TB00249K

75. Crochet J, Clemens M, Hertel T. Quantum yield heterogeneities of aqueous single-wall carbon nanotube suspensions. J Am Chem Soc. 2007;129(26):8058-8059. doi:10.1021/ja071553d

76. Lu G, Shang W, Deng H, et al. Targeting carbon nanotubes based on IGF-1R for photothermal therapy of orthotopic pancreatic cancer guided by optical imaging. Biomaterials. 2019;19 5:13-22. doi:10.1016/j.biomaterials.2018.12.025

77. Sabella S, Carney RP, Brunetti V, et al. A general mechanism for intracellular toxicity of metal-containing nanoparticles. Nanoscale. 2014;6(12):7052-7061. doi:10.1039/c4nr01234h

78. Li X, Yan Y, Lin Y, et al. Hollow mesoporous carbon as a near-infrared absorbing carrier compared with mesoporous carbon nanoparticles for chemophotothermal therapy. $J$ Colloid Interf Sci. 2017;494:159-169. doi:10.1016/j.jcis.2017.01.090

79. Wang X, Lin Y, Li X, et al. Fluorescent carbon dot gated hollow mesoporous carbon for chemo-photothermal synergistic therapy. J Colloid Interf Sci. 2017;507:410-420. doi:10.1016/j.jcis.20 17.08.010

80. Fang J, Liu Y, Chen Y, Ouyang D, Yang G, Yu T. Graphene quantum dots-gated hollow mesoporous carbon nanoplatform for targeting drug delivery and synergistic chemo-photothermal therapy. Int J Nanomed. 2018;13:5991-6007. doi:10.2147/IJN.S175934

81. Wu F, Zhang M, Lu H, et al. Triple stimuli-responsive magnetic hollow porous carbon-based nanodrug delivery system for magnetic resonance imaging-guided synergistic photothermal/chemotherapy of cancer. ACS Appl Mater Inter. 2018;10 (26):21939-21949. doi:10.1021/acsami.8b07213

82. Fan L, Xu X, Zhu C, et al. Tumor catalytic-photothermal therapy with yolk-shell gold@carbon nanozymes. ACS Appl Mater Inter 2018;10(5):4502-4511. doi:10.1021/acsami.7b17916

83. You Q, Sun Q, Wang J, et al. A single-light triggered and dual-imaging guided multifunctional platform for combined photothermal and photodynamic therapy based on TD-controlled and ICG-loaded CuS@mSiO(2). Nanoscale. 2017;9(11):37 84-3796. doi:10.1039/C6NR09042G
84. Xing L, Li X, Xing Z, et al. Silica/gold nanoplatform combined with a thermosensitive gel for imaging-guided interventional therapy in PDX of pancreatic cancer. Chem Eng J. 2020;382:122949. doi:10.1016/j.cej.2019.122949

85. Yu X, Yang K, Chen X, Li W. Black hollow silicon oxide nanoparticles as highly efficient photothermal agents in the second near-infrared window for in vivo cancer therapy. Biomaterials. 2017;143:120-129. doi:10.1016/j.biomaterials.2017.07.037

86. Chen W, Zeng K, Liu H, et al. Cell membrane camouflaged hollow prussian blue nanoparticles for synergistic photothermal-/chemotherapy of cancer. Adv Funct Mater. 2017;27:160579511.

87. Cai X, Gao W, Ma M, et al. A prussian blue-based core-shell hollow-structured mesoporous nanoparticle as a smart theranostic agent with ultrahigh $\mathrm{pH}$-responsive longitudinal relaxivity. $A d v$ Mater. 2015;27(41):6382. doi:10.1002/adma.201503381

88. Song G, Liang C, Yi X, et al. Perfluorocarbon-loaded hollow Bi2Se3 nanoparticles for timely supply of oxygen under near-infrared light to enhance the radiotherapy of cancer. $A d v$ Mater. 2016;28(14):2716-2723. doi:10.1002/adma.201504617

89. Liu Z, Cheng L, Zhang L, Yang Z, Liu Z, Fang J. Sub-100 nm hollow Au-Ag alloy urchin-shaped nanostructure with ultrahigh density of nanotips for photothermal cancer therapy. Biomaterials. 2014;35(13):4099-4107. doi:10.1016/j.biomaterials. 2014.01.053

90. Li J, Zhang F, Hu Z, et al. Drug "pent-up" in hollow magnetic prussian blue nanoparticles for nir-induced chemo-photothermal tumor therapy with trimodal imaging. Adv Healthc Mater. 2017;6:170000514.

91. Ungelenk S, Moayed F, Ho C, et al. Small heat shock proteins sequester misfolding proteins in near-native conformation for cellular protection and efficient refolding. Nat Commun. 2016;7:13673.

92. Chen W, Luo G, Lei Q, et al. Overcoming the heat endurance of tumor cells by interfering with the anaerobic glycolysis metabolism for improved photothermal therapy. Acs Nano Nano. 2017;11 (2):1419-1431. doi:10.1021/acsnano.6b06658

93. Jiang Y, Li J, Zhen X, Xie C, Pu K. Dual-peak absorbing semiconducting copolymer nanoparticles for first and second near-infrared window photothermal therapy: a comparative study. Adv Mater. 2018;30:170598014. doi:10.1002/adma.201705980

94. Zhang $\mathrm{N}$, Han $\mathrm{C}, \mathrm{Xu} \mathrm{Y}$, et al. Near-field dielectric scattering promotes optical absorption by platinum nanoparticles. Nat Photonics. 2016;10(7):473-482. doi:10.1038/nphoton.2016.76

95. Wang Q, Wang H, Yang Y, et al. Plasmonic Pt superstructures with boosted near-infrared absorption and photothermal conversion efficiency in the second biowindow for cancer therapy. $A d v$ Mater. 2019;31:190483646. doi:10.1002/adma.201904836

96. Mohan R. Green bismuth. Nat Chem. 2010;2(4):336. doi:10.1038/ nchem.609

97. Huang Y, Xue Z, Zeng S. Hollow mesoporous Bi@PEG-FA nanoshell as a novel dual-stimuli-responsive nanocarrier for synergistic chemo-photothermal cancer therapy. ACS Appl Mater Inter. 2020;12(28):31172-31181. doi:10.1021/acsami.0c07372

98. Song XR, Yu SX, Jin GX, et al. Plant polyphenol-assisted green synthesis of hollow CoPt alloy nanoparticles for dual-modality imaging guided photothermal therapy. Small. 2016;12(11): 1506-1513. doi:10.1002/smll.201503250

99. Xu M, Lu Q, Song Y, Yang L, Li J, Li N. Enhanced Bax upregulating in mitochondria for deep tumor therapy based on $\mathrm{SO}(2)$ prodrug loaded Au-Ag hollow nanotriangles. Biomaterials. 2020;250:120076. doi:10.1016/j.biomaterials.20 20.120076

100. Meeker DG, Jenkins SV, Miller EK, et al. Synergistic photothermal and antibiotic killing of biofilm-associated staphylococcus aureus using targeted antibiotic-loaded gold nanoconstructs. ACS Infect Dis. 2016;2(4):241-250. doi:10.1021/acsinfecdis.5b00117 
101. Meeker DG, Wang T, Harrington WN, et al. Versatility of targeted antibiotic-loaded gold nanoconstructs for the treatment of biofilm-associated bacterial infections. Int J Hyperther. 2018;34 (2):209-219. doi:10.1080/02656736.2017.1392047

102. He J, Qiao Y, Zhang H, et al. Gold-silver nanoshells promote wound healing from drug-resistant bacteria infection and enable monitoring via surface-enhanced Raman scattering imaging. Biomaterials. 2020;234:119763. doi:10.1016/j.biomaterials.2020. 119763

103. Xuan M, Shao J, Dai L, Li J, He Q. Macrophage cell membrane camouflaged Au nanoshells for in vivo prolonged circulation life and enhanced cancer photothermal therapy. ACS Appl Mater Interfaces. 2016;8(15):9610-9618. doi:10.1021/ acsami.6b00853

104. Kawai T, Akira S. Toll-like receptors and their crosstalk with other innate receptors in infection and immunity. Immunity. 2011;34(5):637-650. doi:10.1016/j.immuni.2011.05.006

105. Wang C, Wang Y, Zhang L, et al. Pretreated macrophage-membrane-coated gold nanocages for precise drug delivery for treatment of bacterial infections. Adv Mater. 2018;30 (46):e1804023. doi:10.1002/adma.201804023

106. Pihlstrom BL, Michalowicz BS, Johnson NW. Periodontal diseases. Lancet. 2005;366(9499):1809-1820. doi:10.1016/ S0140-6736(05)67728-8

107. Nazir MA. Prevalence of periodontal disease, its association with systemic diseases and prevention. Int $J$ Health Sci (Qassim). 2017;11(2):72-80.

108. Zhang L, Wang Y, Wang C, et al. Light-activable on-demand release of nano-antibiotic platforms for precise synergy of thermochemotherapy on periodontitis. ACS Appl Mater Interfaces. 2020;12(3):3354-3362. doi:10.1021/acsami.9b17335

109. Hu B, Zhang LP, Chen XW, Wang JH. Gold nanorod-covered kanamycin-loaded hollow SiO2 (HSKAu(rod)) nanocapsules for drug delivery and photothermal therapy on bacteria. Nanoscale. 2013;5(1):246-252. doi:10.1039/C2NR32457A

110. Wu S, Li A, Zhao X, et al. Silica-coated gold-silver nanocages as photothermal antibacterial agents for combined anti-infective therapy. ACS Appl Mater Interfaces. 2019;11(19):17177-17183. doi:10.1021/acsami.9b01149

111. Hynes WL, Walton SL. Hyaluronidases of gram-positive bacteria. FEMS Microbiol Lett. 2000;183(2):201-207. doi:10.1111/j.15746968.2000.tb08958.x

112. Choi KY, Yoon HY, Kim J, et al. Smart nanocarrier based on PEGylated hyaluronic acid for cancer therapy. Acs Nano Nano. 2011;5(11):8591-8599. doi:10.1021/nn202070n

113. Lee Y, Lee H, Kim YB, et al. Bioinspired surface immobilization of hyaluronic acid on monodisperse magnetite nanocrystals for targeted cancer imaging. Adv Mater. 2008;20(21):4154.

114. Ren J, Zhang L, Zhang J, et al. Light-activated oxygen self-supplied starving therapy in near-infrared (NIR) window and adjuvant hyperthermia-induced tumor ablation with an augmented sensitivity. Biomaterials. 2020;234:119771. doi:10.1016/ j.biomaterials.2020.119771

115. Sun L, Li Q, Zhang L, et al. Stimuli responsive PEGylated bismuth selenide hollow nanocapsules for fluorescence/CT imaging and light-driven multimodal tumor therapy. Biomater Sci. 2019;7(7):3025-3040. doi:10.1039/C9BM00351G

116. Wang $\mathrm{W}$, Zhang $\mathrm{C}$, Zhang M, et al. Precisely photothermal controlled releasing of antibacterial agent from Bi2S3 hollow microspheres triggered by NIR light for water sterilization. Chem Eng J. 2020;381: 122630. doi:10.1016/j.cej.2019.122630

117. Qiao Y, He J, Chen W, et al. Light-activatable synergistic therapy of drug-resistant bacteria-infected cutaneous chronic wounds and nonhealing keratitis by cupriferous hollow nanoshells. Acs Nano Nano. 2020;14(3):3299-3315. doi:10.1021/acsnano.9b08930
118. Ye Y, He J, Qiao Y, et al. Mild temperature photothermal assisted anti-bacterial and anti-inflammatory nanosystem for synergistic treatment of post-cataract surgery endophthalmitis. Theranostics. 2020;10(19):8541-8557. doi:10.7150/thno.46895

119. Rodriguez-Sevilla P, Zhang Y, Haro-Gonzalez P, et al. Thermal scanning at the cellular level by an optically trapped upconverting fluorescent particle. Adv Mater. 2016;28(12):2421-2426. doi:10. 1002/adma.201505020

120. Del Rosal B, Ximendes E, Rocha U, Jaque D. In vivo luminescence nanothermometry: from materials to applications. $A d v O p t$ Mater. 2017;5(1):16005081. doi:10.1002/adom.201600508

121. Suo H, Zhao X, Zhang Z, Guo C. $808 \mathrm{~nm}$ light-triggered thermometer-heater upconverting platform based on $\mathrm{Nd}(3+)--$ sensitized yolk-shell GdOF@SiO(2). ACS Appl Mater Interfaces. 2017;9(50):43438-43448. doi:10.1021/acsami.7b12753

122. Suo H, Zhao X, Zhang Z, Wu Y, Guo C. Upconverting LuVO(4):Nd(3 $+) / \mathrm{Yb}(3+) / \mathrm{Er}(3+) @ \mathrm{SiO}(2) @ \mathrm{Cu}(2) \mathrm{S}$ hollow nanoplatforms for self-monitored photothermal ablation. ACS Appl Mater Interfaces. 2018;10(46):39912-39920. doi:10.1021/acsami.8b18184

123. Yu P, Han Y, Han D, et al. In-situ sulfuration of Cu-based metal-organic framework for rapid near-infrared light sterilization. $J$ Hazard Mater. 2020;390:122126. doi:10.1016/j.jhazmat.2020.122126

124. Blennow K, de Leon MJ, Zetterberg H. Alzheimer's disease. Lancet. 2006;368(9533):387-403. doi:10.1016/S0140-6736(06)69113-7

125. Prince M, Bryce R, Albanese E, Wimo A, Ribeiro W, Ferri CP. The global prevalence of dementia: a systematic review and metaanalysis. Alzheimers Dement. 2013;9(1):63-75. doi:10.1016/j.jalz.2012.11.007

126. Selkoe DJ. Alzheimer's disease: genes, proteins, and therapy. Physiol Rev. 2001;81(2):741-766. doi:10.1152/physrev.2001. 81.2.741

127. Poduslo JF, Curran GL, Kumar A, Frangione B, Soto C. Betasheet breaker peptide inhibitor of Alzheimer's amyloidogenesis with increased blood-brain barrier permeability and resistance to proteolytic degradation in plasma. $J$ Neurobiol. 1999;39 (3):371-382. doi:10.1002/(SICI)1097-4695(19990605)39:3<371:: AID-NEU4>3.0.CO;2-E

128. Hudson SA, Ecroyd H, Dehle FC, Musgrave IF, Carver JA. (-)epigallocatechin-3-gallate (EGCG) maintains kappa-casein in its pre-fibrillar state without redirecting its aggregation pathway. J Mol Biol. 2009;392(3):689-700. doi:10.1016/j.jmb.2009.07.031

129. Cabaleiro-Lago C, Quinlan-Pluck F, Lynch I, et al. Inhibition of amyloid beta protein fibrillation by polymeric nanoparticles. $\mathrm{J} \mathrm{Am}$ Chem Soc. 2008;130(46):15437-15443. doi:10.1021/ja8041806

130. Ruff J, Hüwel S, Kogan MJ, Simon U, Galla HJ. The effects of gold nanoparticles functionalized with $B$-amyloid specific peptides on an in vitro model of blood-brain barrier. NanomedicineUk. 2017;13(5):1645-1652. doi:10.1016/j.nano.2017.02.013

131. Ruff J, Hassan N, Morales-Zavala F, et al. CLPFFD-PEG functionalized NIR-absorbing hollow gold nanospheres and gold nanorods inhibit $\beta$-amyloid aggregation. $J$ Mater Chem $B$. 2018;6(16):2432-2443. doi:10.1039/C8TB00655E

132. Guerrero S, Herance JR, Rojas S, et al. Synthesis and in vivo evaluation of the biodistribution of a 18F-labeled conjugate gold-nanoparticle-peptide with potential biomedical application. Bioconjug Chem. 2012;23(3):399-408. doi:10.1021/bc200362a

133. Guerrero S, Araya E, Fiedler JL, et al. Improving the brain delivery of gold nanoparticles by conjugation with an amphipathic peptide. Nanomedicine (Lond). 2010;5(6):897-913. doi:10.2217/nnm.10.74

134. Alavi NS, Soussi-Yanicostas N. Tau hyperphosphorylation and oxidative stress, a critical vicious circle in neurodegenerative tauopathies? Oxid Med Cell Longev. 2015;2015:151979.

135. Zhou H, Gong Y, Liu Y, et al. Intelligently thermoresponsive flowerlike hollow nano-ruthenium system for sustained release of nerve growth factor to inhibit hyperphosphorylation of tau and neuronal damage for the treatment of Alzheimer's disease. Biomaterials. 2020;237:119822. doi:10.1016/j.biomaterials.2020.119822 
136. Chan RS, Woo J. Prevention of overweight and obesity: how effective is the current public health approach. Int J Environ Res Public Health. 2010;7(3):765-783. doi:10.3390/ijerph7030765

137. $\mathrm{Ng} \mathrm{M}$, Fleming $\mathrm{T}$, Robinson $\mathrm{M}$, et al. Global, regional, and national prevalence of overweight and obesity in children and adults during 1980-2013: a systematic analysis for the Global Burden of Disease Study 2013. Lancet. 2014;384(9945):766-781. doi:10.1016/S0140-6736(14)60460-8

138. Mitchell AB, Cole JW, McArdle PF, et al. Obesity increases risk of ischemic stroke in young adults. Stroke. 2015;46 (6):1690-1692. doi:10.1161/STROKEAHA.115.008940

139. McCarthy MI, Feero WG, Guttmacher AE. Genomics, type 2 diabetes, and obesity. $N$ Engl J Med. 2010;363(24):2339-2350. doi:10.1056/NEJMra0906948

140. Fabbrini E, Sullivan S, Klein S. Obesity and nonalcoholic fatty liver disease: biochemical, metabolic, and clinical implications. Hepatology. 2010;51(2):679-689. doi:10.1002/hep.23280

141. Li J, Cha R, Luo H, Hao W, Zhang Y, Jiang X. Nanomaterials for the theranostics of obesity. Biomaterials. 2019;223:119474. doi:10.1016/j.biomaterials.2019.119474

142. Han S, Kim Y. Polypyrrole-coated hollow gold nanoshell exerts anti-obesity effects via photothermal lipolysis. Coll Surf A 2019;570:414-419. doi:10.1016/j.colsurfa.2019.03.063

143. Lee JH, Jeong HS, Lee DH, et al. Targeted hyaluronate-hollow gold nanosphere conjugate for anti-obesity photothermal lipolysis. ACS Biomater Sci Eng. 2017;3(12):3646-3653. doi:10.1021/acsbiomaterials.7b00549

144. Vercellini P, Viganò P, Somigliana E, Fedele L. Endometriosis: pathogenesis and treatment. Nat Rev Endocrinol. 2014;10 (5):261-275. doi:10.1038/nrendo.2013.255

145. Wilson ML, Fleming KA, Kuti MA, Looi LM, Lago N, Ru K. Pathology and laboratory medicine in low-income and middle-income countries 1: access to pathology and laboratory medicine services: a crucial gap. Lancet. 2018;391 (10133):1927-1938. doi:10.1016/S0140-6736(18)30458-6

146. Eskenazi B, Warner ML. Epidemiology of endometriosis. Obstet Gynecol Clin North Am. 1997;24(2):235-258. doi:10.1016/ S0889-8545(05)70302-8
147. Kyama CM, Overbergh L, Mihalyi A, et al. Endometrial and peritoneal expression of aromatase, cytokines, and adhesion factors in women with endometriosis. Fertil Steril. 2008;89 (2):301-310. doi:10.1016/j.fertnstert.2007.02.057

148. Burney RO, Giudice LC. Pathogenesis and pathophysiology of endometriosis. Fertil Steril. 2012;98(3):511-519. doi:10.1016/j. fertnstert.2012.06.029

149. Kikuchi I, Takeuchi H, Kitade M, Shimanuki H, Kumakiri J, Kinoshita K. Recurrence rate of endometriomas following a laparoscopic cystectomy. Acta Obstet Gyn Scan. 2006;85 (9):1120-1124. doi:10.1080/00016340600627154

150. Oral E, Aydin O, Kumbak BA, et al. Concomitant endometriosis in malignant and borderline ovarian tumours(*). J Obstet Gynaecol. 2018;38(8):1104-1109. doi:10.1080/01443615.2018. 1441815

151. Guo X, Li W, Zhou J, et al. Specific photothermal ablation therapy of endometriosis by targeting delivery of gold nanospheres. Small. 2017;13(15):1603270. doi:10.1002/sm11.20 1603270

152. Kharlamov AN, Tyurnina AE, Veselova VS, Kovtun OP, Shur VY, Gabinsky JL. Silica-gold nanoparticles for atheroprotective management of plaques: results of the NANOM-FIM trial. Nanoscale. 2015;7(17):8003-8015. doi:10.1039/C5NR01050K

153. Kharlamov AN, Feinstein JA, Cramer JA, Boothroyd JA, Shishkina EV, Shur V. Plasmonic photothermal therapy of atherosclerosis with nanoparticles: long-term outcomes and safety in NANOM-FIM trial. Future Cardiol. 2017;13(4):345-363. doi:10.2217/fca-2017-0009

154. Gad SC, Sharp KL, Montgomery C, Payne JD, Goodrich GP. Evaluation of the toxicity of intravenous delivery of auroshell particles (gold-silica nanoshells). Int $J$ Toxicol. 2012;31 (6):584-594. doi:10.1177/1091581812465969

155. Hu Y, Chi C, Wang S, et al. A comparative study of clinical intervention and interventional photothermal therapy for pancreatic cancer. Adv Mater. 2017;29(33):1700448. doi:10.1002/adma. 201700448
International Journal of Nanomedicine

\section{Publish your work in this journal}

The International Journal of Nanomedicine is an international, peerreviewed journal focusing on the application of nanotechnology in diagnostics, therapeutics, and drug delivery systems throughout the biomedical field. This journal is indexed on PubMed Central, MedLine, CAS, SciSearch ${ }^{\mathbb{}}$, Current Contents ${ }^{\circledR} /$ Clinical Medicine, $^{2}$
Journal Citation Reports/Science Edition, EMBase, Scopus and the Elsevier Bibliographic databases. The manuscript management system is completely online and includes a very quick and fair peer-review system, which is all easy to use. Visit http://www.dovepress.com/ testimonials.php to read real quotes from published authors. 